\title{
Magnetic fields and gas in the cluster-influenced spiral galaxy NGC 4254
}

\section{Structures of magnetic fields}

\author{
K. T. Chyży \\ Astronomical Observatory, Jagiellonian University, ul. Orla 171, 30-244 Kraków, Poland \\ e-mail: chris@oa.uj.edu.pl
}

Received 17 September 2007 / Accepted 4 February 2008

\begin{abstract}
Aims. The origin of asymmetric radio polarized emission in the Virgo Cluster spiral NGC 4254 is investigated and the influence of cluster environment on the properties of magnetic fields is explored.

Methods. Structures of magnetic fields are analyzed with the concept of "magnetic maps", presenting distributions of different magnetic field components (total, regular, and random) over the entire galaxy, free of Faraday rotation and projection effects. A number of different physical phenomena influencing the magnetic field are modeled analytically and confronted with the galaxy's depolarization pattern and distribution of magnetic field strength obtained from multifrequency polarimetric radio observations.

Results. The study of orientation of intrinsic magnetic field vectors in NGC 4254 indicates that their dramatic variation (from $0^{\circ}$ to more than $40^{\circ}$ ) throughout the galaxy cannot arise from the dynamo process alone, but must be dominated by effects such as density waves and local gas flows. We determine within the galaxy the relation between the strength of total magnetic field and the local star-formation rate (SFR) as a power-law with an index of $+0.18 \pm 0.01$. We find the opposite sense of the relation between magnetic field regularity and SFR $(-0.32 \pm 0.03)$, and suggest that it results from efficient production of random field with rising turbulence in the regions with actively-forming stars. The distribution of Faraday rotation measures in NGC 4254 indicates a perturbed axisymmetrical mean-field dynamo mode or a mixture of axisymmetrical and bisymmetrical ones with regular field directed outwards from the disk, which is contrary to most observed galaxies. The galaxy's northern magnetic arm, located on the upstream side of the local density wave, with regular field strength of about $8 \mu \mathrm{G}$ and the total one of $17 \mu \mathrm{G}$, much resembles those observed in other galaxies. But the magnetic field within two outer arms (shifted downstream of a density wave) is much stronger, up to $13 \mu \mathrm{G}$ in the regular field component and $20 \mu \mathrm{G}$ in the total field. Our modeling of cluster influence on different magnetic field components indicates that within the outer magnetic arms the dynamo-induced magnetic fields are modified by stretching and shearing forces rather than by cluster ram pressure. Those forces, which are likely triggered by the galaxy's gravitational interaction, produce an anisotropic component of the regular field and enhance the polarized emission. We also show that the magnetic energy within the large interarm regions and the galaxy's outskirts exceeds the gas thermal and turbulent energy, likely becoming dynamically important.
\end{abstract}

Key words. galaxies: general - ISM: magnetic fields - galaxies: magnetic fields - galaxies: interactions radio continuum: galaxies - radio continuum: ISM

\section{Introduction}

Disk galaxies in galaxy clusters are particularly affected by the cluster environment (Roediger \& Hensler 2005) and can be even entirely transformed from one Hubble type to another (Moore et al. 1996). Interstellar medium (ISM) within a cluster galaxy is altered in a different manner in individual galactic regions, as can be seen, e.g., from $\mathrm{H} \alpha$ (Koopmann \& Kenney 2004) and H I (Cayatte et al. 1990) distributions. The magnetic field in cluster galaxies as one of the ISM ingredients is also expected to be significantly modified, mainly by ram-pressure (compression) of hot intracluster medium (ICM) or by tidal (stretching/shearing) forces due to galactic encounters. Investigation of influence of such external forces on magnetic field gives an independent insight into the galaxy evolution within the cluster (Soida et al. 2006). Exploring magnetic field structures can also allow for determining how tangling of magnetic field within star-forming regions alters different magnetic field components, or to what extent the gaseous streamlines perturbed in cluster galaxies can tune the dynamo-induced galactic magnetic fields (Shukurov 1998; Elstner 2005). To trace all those processes locally, we need sensitive radio polarimetric data of at least kpc-scale resolution, which are available now only for the Virgo Cluster spirals.

In this paper we present the first detailed analysis of magnetic field, rotation measure, and depolarization patterns of a cluster spiral - the perturbed Virgo Cluster galaxy NGC 4254. In Paper I (Chyży et al. 2007) we presented our radio polarimetric observations (of the VLA of $\mathrm{NRAO}^{1}$ and 100-m Effelsberg telescope $^{2}$ ) and X-rays observations (from XMM-Newton ${ }^{3}$ ),

\footnotetext{
1 National Radio Astronomy Observatory is a facility of National Science Foundation operated under a cooperative agreement by Associated Universities, Inc.

2 The 100-m telescope at Effelsberg is operated by the Max-PlanckInstitut für Radioastronomie (MPIfR) on behalf of the Max-PlanckGesellschaft.

3 XMM-Newton is an ESA science mission with instruments and contributions directly funded by ESA Member States and NASA (Jansen et al. 2001).
} 


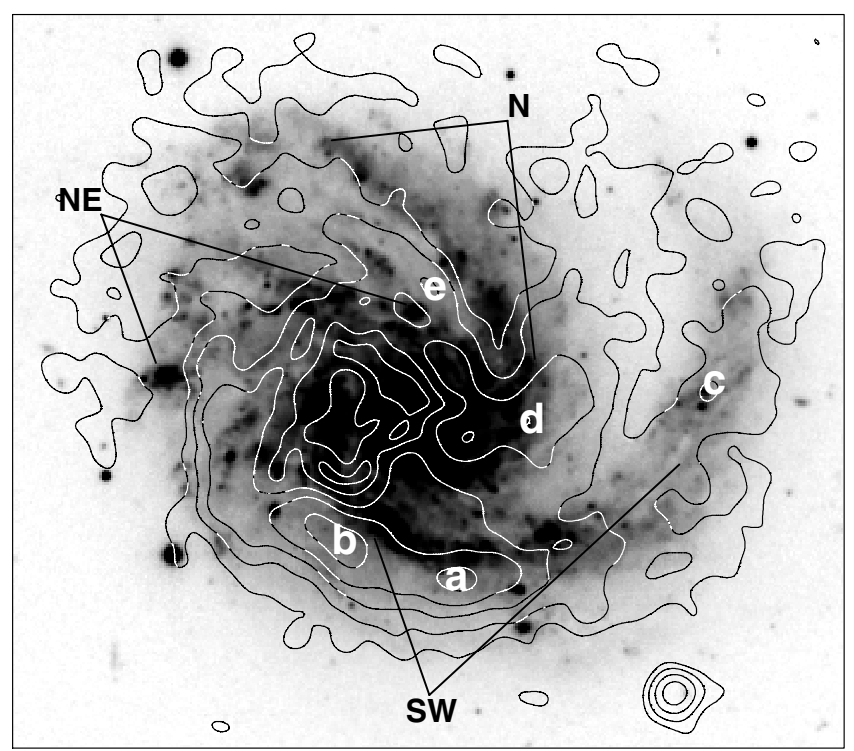

Fig. 1. Optical DSS B image of NGC 4254 and polarized intensity at $4.86 \mathrm{GHz}$ in $15^{\prime \prime}$ resolution in contours. The contours are at $9.7 \times$ $(4,9,16,25,40) \mu \mathrm{G}$. Three main optical spiral arms (SW, N and NE) are schematically marked. Letters a-e denote positions of contrast derivations in Sect. 3.5.

together with the data reduction process, as well as a global outline of the galaxy's radio and polarimetric properties in relation to other spectral bands. We found that in the radio domain the galaxy has an asymmetrical distribution extending to the north, which resembles disturbed distributions in the optical, $\mathrm{UV}$, and X-rays bands, dominated by a one-arm spiral structure. The polarized intensity maps at $8.46 \mathrm{GHz}$ and $4.86 \mathrm{GHz}$ show an unusual strong ridge in the southern disk portion, outside of a heavy optical spiral arm, with observed magnetic field vectors directed along it (Fig. 1, see also Fig. 2 in Paper I). Similar, but weaker, structures shifted off optical features are also visible in other parts of the galaxy disk. Some polarized features are strong enough to appear within optical filaments even in the highly turbulent spiral arm regions. These mixed magnetic field patterns are in sharp contrast to the previous studied grand-design spirals, which typically show either interarm or arm-dominated polarized structures (like in NGC 6946 - Beck \& Hoernes 1996 and M 51 - Fletcher et al. 2004b, respectively). Moreover, the star-formation rate (SFR) derived from radio thermal emission is higher in NGC 4254 than in other galaxies, indicating some effects of an external (cluster) agent.

The pattern of magnetic fields discovered in NGC 4254 is investigated in the present paper in a detailed manner. In Sect. 2.1, we present the Faraday rotation measure and depolarization maps and derive a Faraday-free intrinsic structure of magnetic field. Next, we introduce "magnetic maps" (Sect. 2.3) - a novel concept of presenting magnetic field strength in different magnetic field components in the form of distributions across the entire galaxy, free of Faraday rotation and projection effects. In Sect. 3.1, we study the orientation (pitch angle) of magnetic field vectors within structures of optical arms and gas filaments. We investigate regularity of magnetic field over regions of different star formation rates (Sect. 3.2) and model various Faraday depolarization effects (Sect. 3.3). The impact of external compression and stretching effects on the different components of galactic magnetic field is analytically modeled in Sect. 3.4 and finally discussed in Sect. 3.5.

\section{Results}

\subsection{Distribution of Faraday rotation and depolarization}

For the purpose of analyzing magnetic field in NGC 4254 we use the radio polarimetric observations described in detail in Paper I. The observed polarized component of synchrotron emission results from the regular (ordered) component of magnetic field. It can contain a mixture of coherent (unidirectional) and anisotropic (incoherent) magnetic fields. Only the coherent field contributes to the observed Faraday rotation because the contributions from an anisotropic field with vectors of opposite directions cancel when averaged over the telescope beam. To construct the Faraday rotation measure $(R M)$ map we take the distributions of polarization angles at 4.86 and $8.46 \mathrm{GHz}$ in $15^{\prime \prime}$ resolution. The observed Faraday rotation also includes the contribution from the Galaxy's foreground rotation measure. The spiral NGC 4254 lies at $270.4^{\circ}$ Galactic longitude and $75.2^{\circ}$ Galactic latitude, where Johnston-Hollitt et al. (2004) show only low values of foreground $R M$, changing sign from place to place with absolute values $<25 \mathrm{rad} \mathrm{m}^{-2}$. The average value of $R M$ estimated from our data over the entire galaxy is also small: about $+15 \mathrm{rad} \mathrm{m}^{-2}$. Some reliable measure of foreground $R M$ can be obtained from a polarized background source visible to the SW from NGC $4254\left(\mathrm{RA}_{2000}=12^{\mathrm{h}} 18^{\mathrm{m}} 42^{\mathrm{s}} \cdot 1, \operatorname{Dec}_{2000}=\right.$ $+14^{\circ} 23^{\prime} 07^{\prime \prime}$, Fig. 2a). Within the uncertainties in measurement of about $3^{\circ}$, the source shows no difference in polarization position angle between 8.46 and $4.86 \mathrm{GHz}$, thus suggesting a low $R M\left(<20 \mathrm{rad} \mathrm{m}^{-2}\right)$. Between 4.86 and $1.43 \mathrm{GHz}$ we measured the position angle difference of $5.5^{\circ}$, which provides very small $R M$ of $2.5 \mathrm{rad} \mathrm{m}^{-2}$. Therefore, we do not apply any correction to the data for the foreground Faraday rotation.

The $R M$ map for NGC 4254 is presented in Fig. 2a together with contours of total radio emission. Over many galactical regions the absolute values of $R M$ are typically small, of the order of $50-70 \mathrm{rad} \mathrm{m}^{-2}$ and exceed $100 \mathrm{rad} \mathrm{m}^{-2}$ only within small regions. The variation of $R M$ over the disk can reflect various physical processes (see Sect. 2.1) and local changes of magnetic field strength as well as its orientation with respect to the observer. In general, the western part of the galaxy has negative $R M$ values, while the eastern part has positive ones that are slightly larger in the absolute sense. This demonstrates a substantial coherence of the sign of $R M$ and hence of the magnetic field orientation. Such coherence of magnetic field strongly suggests a global galactic dynamo in action (Beck et al. 1996; Widrow 2002).

Close to the center of NGC 4254 there are two sudden jumps of $R M$ oriented at $90^{\circ}$ to each other $\left(\mathrm{RA}=12^{\mathrm{h}} 18^{\mathrm{m}} 51^{\mathrm{s}} \cdot 0\right.$, Dec $=$ $+14^{\circ} 24^{\prime} 31^{\prime \prime}$; and RA $=12^{\mathrm{h}} 18^{\mathrm{m}} 52^{\mathrm{s}} \cdot 0$, Dec $\left.=+14^{\circ} 25^{\prime} 20^{\prime \prime}\right)$. In these places, our map keeps track of $R M$ values up to about $\pm 400 \mathrm{rad} \mathrm{m}^{-2}$. Such jumps usually mean that the internal Faraday rotation angle exceeds $90^{\circ}$ and indicate a Faraday-thick regime (Sokoloff et al. 1998). The jumps do not correspond to any feature in the radio or H I emission. However, the southern jump is close to a $\mathrm{CO}$ extension going radially outwards from the nuclear ring (see Fig. 3 in Sofue et al. 2003).

The region of the SW optical arm where the strongest polarized emission in NGC 4254 is observed shows a rather small amount of Faraday rotation: around its eastern polarized peak (marked as "a" in Fig. 1) the $R M$ is about $-30 \pm 7 \mathrm{radm}^{-2}$. Around the western peak (marked as "b" in Fig. 1) the $R M$ has the opposite sign and a value of about $+20 \pm 6 \mathrm{rad} \mathrm{m}^{-2}$. This may indicate a change of magnetic field orientation with respect to the observer. In fact, the two peaks are on both sides of the galaxy's minor axis (see Fig. 2a with marked the galaxy's major axis at 

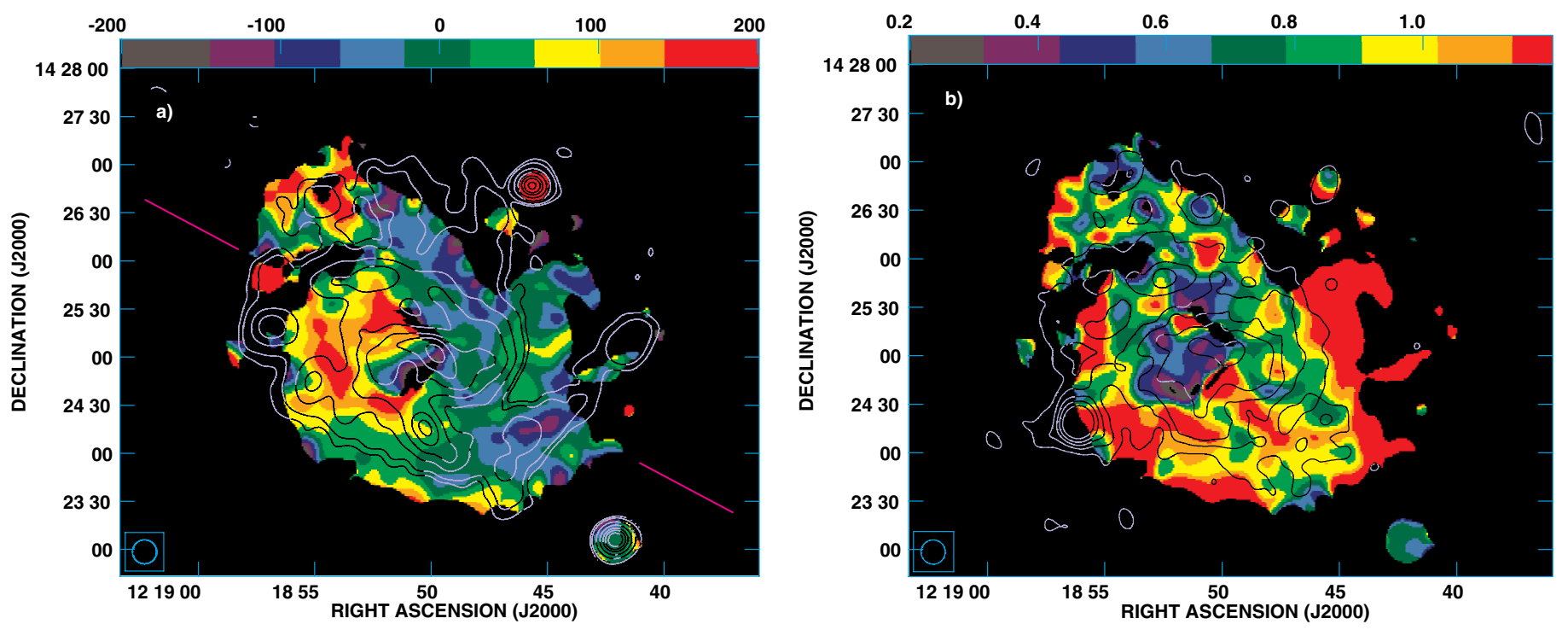

Fig. 2. a) Faraday rotation measure distribution in NGC 4254 in $15^{\prime \prime}$ resolution in colors, computed between $8.46 \mathrm{GHz}$ and $4.86 \mathrm{GHz}$ with contours of total radio intensity at $4.86 \mathrm{GHz}$. The galaxy major axis is marked. b) Faraday depolarization distribution in colors and contours of soft X-ray emission from our XMM-Newton observations.

the position angle of $68^{\circ}$ envisaged from the $\mathrm{H}$ I gas kinematics, Phookun et al. 1993).

Faraday rotation effects not only change the orientation of actual magnetic field vectors but also modify (typically reduce) the observed degree of polarization. We measure the wavelength-dependent depolarization $D P$ as the ratio of the degree of polarization $(p)$ of nonthermal emission at $4.86 \mathrm{GHz}$ $(6.2 \mathrm{~cm})$ and $8.46 \mathrm{GHz}(3.5 \mathrm{~cm})$ :

$D P=p_{4.86} / p_{8.46}$

We separated the nonthermal emission at both frequencies from the total radio intensity with our observations at $1.43 \mathrm{GHz}$, $4.86 \mathrm{GHz}$, and $8.46 \mathrm{GHz}$, assuming a constant nonthermal spectral index $\alpha_{\text {nth }}=1.0$ (cf. Paper I). The map of depolarization of NGC 4254 presented in Fig. 2b exhibits globally an asymmetric pattern, as in the case of the $R M$ map. The southern and western parts of the galaxy show very little depolarization $(D P>0.9)$. This is in agreement with substantially-polarized emission observed here at a low frequency of $1.43 \mathrm{GHz}$ (Paper I), which is an independent indication of Faraday-thin regime.

In the eastern galaxy part depolarization is stronger, with $D P$ values of about $0.6-0.8$. The strongest depolarization effects occur at the nucleus, where locally $D P \approx 0.2$, and around $R M$ jumps, where $D P<0.2$. These low $D P$ values must have been produced by a strong Faraday rotation within the emitting region (Sokoloff et al. 1998 and Sect. 3.3). All those central regions are actually associated with strong $\mathrm{H} \alpha$ and hot gas (soft X-ray) emissions. As expected, they also show rather weak polarized intensity at $1.43 \mathrm{GHz}$ (less than $0.03 \mathrm{mJy}$ ) and a weak degree of polarization $(<1 \%)$.

We attempt to identify main processes to affect polarization in NGC 4254 by performing a mutual comparison between $D P$, $R M$, and radio thermal emission in 136 beam-independent regions within the galaxy. There is no statistically important global correlation of the thermal gas emission with the $D P$ or $R M$ values: the Pearson correlation coefficient $r$ is around -0.10 . The relation of $D P$ with $R M$ is also weak $(r=-0.02)$. Thus, no single process associated with the galactic thermal gas or the regular magnetic field is capable of accounting for the $D P$ distribution. Therefore, a more detailed analysis in separate regions in the galaxy is needed (see Sect. 3.3).

\subsection{Genuine structure of magnetic field}

The same sign of $R M$ values over large regions in NGC 4254 (Sect. 2.1, Fig. 2a) most probably originates from a dynamogenerated magnetic field. In order to recognize a dominant dynamo mode in action we determined a distribution of $R M$ within the galactic plane along rings around the disk center. The rings of radial width of $1.2 \mathrm{kpc}$ (corresponding to the beam size of $15^{\prime \prime}$ ) were split along azimuthal angle into sectors of $15^{\circ}$ width. For each sector we calculated the average rotation measure and we present the distribution of $R M$ in five rings in Fig. 3. Then, in the most characteristic rings 3 and 4 , encompassing the southern polarized ridge, we fit different periodic variations of $R M$ resulting from dynamo modes and their superpositions. The best fits are presented in Table 1 and Fig. 3. The $R M$ distributions partly resemble single-periodic variations, thus the axisymmetric $m=0$ mode. The fitted phase shifts also correspond well to the observed magnetic pitch angles (about $20^{\circ}$ ) in the first and the second quadrant of the azimuthal angle. However, a strong disturbance breaks the dynamo symmetry in the third quadrant (the azimuth in the range of $150^{\circ}-270^{\circ}$ ). The $R M$ instead of achieving, as predicted by the model, a negative minimum of about $-200 \mathrm{rad} \mathrm{m}^{-2}$ manifests a more complicated pattern involving a local field reversal. This deviation corresponds to a strongly-polarized large interarm region between SW/N optical arms, with a quite smooth magnetic field pattern. Similarly, the bisymmetric $(m=1)$ dynamo mode alone cannot account for the $R M$ distribution as it yields too strong a maximum at azimuth of $210^{\circ}$ and too weak one at $40^{\circ}$. However, adding it as a secondary component to the axisymmetric mode (with roughly half of its amplitude) significantly improves the fits. Higher dynamo modes and other mixed modes do not provide any better fits. Surprisingly, it is also true for a quadrupolar $(m=2)$ mode and a mixture $m=0 / 2$ modes, which were suggested to explain the phenomena of magnetic arms in NGC 6946 (Beck 2007; Rohde et al. 1999). 


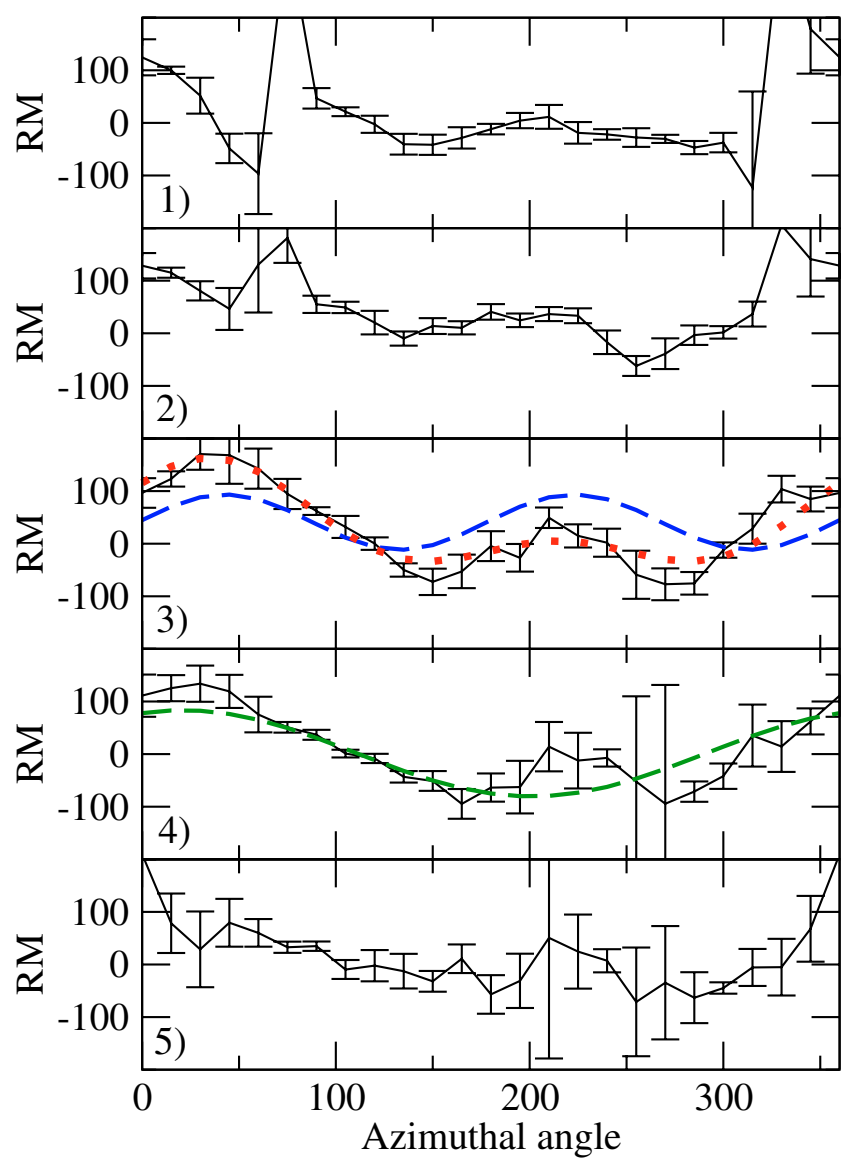

Fig. 3. Distribution of rotation measures between 8.46 and $4.86 \mathrm{GHz}$ along the azimuthal angle in 5 different rings of NGC 4254 . The rings of $1.2 \mathrm{kpc}$ width start from $30^{\prime \prime}(2.4 \mathrm{kpc})$ distance from the galaxy center (ring 1, the most upper one). The last ring (bottom) ends at distance of $8.5 \mathrm{kpc}$. The best-fitted dynamo modes are also shown: for the ring 3 the bisymmetric $m=1$ mode (dashed) and superposition of axisymmetric and bisymmetric $m=0 / 1$ modes (dotted); and for ring 4 the axisymmetric $m=0$ mode (dashed), see Table 1 .

Table 1. Parameters of the single $(m=0)$ double periodic $(m=1)$ cosine functions and their superposition $(m=0 / 1)$ fitted to the rotation measure variations in the two rings of NGC 4254 (cf. Fig. 3). Amplitude, phase shift, background offset, and the Theil U coefficient of the quality of fit are given.

\begin{tabular}{lrrrc}
\hline \hline Dynamo mode & Ampl. & Ph. shift & Offset & ${\text { Theil } \mathrm{U}^{a}}$ \\
\hline RING 3 & & & & \\
$m=0$ & 79 & 25 & 23 & 0.58 \\
$m=1$ & 53 & 43 & 41 & 0.83 \\
$m=0 / 1$ & $79 / 51$ & 34 & 33 & 0.34 \\
\hline RING 4 & & & & \\
$m=0$ & 82 & 21 & 2 & 0.57 \\
$m=1$ & 48 & 48 & 17 & 0.94 \\
$m=0 / 1$ & $71 / 36$ & 33 & 9 & 0.36 \\
\hline
\end{tabular}

${ }^{a}$ The Theil $\mathrm{U}$ coefficient is limited to a range from zero to one (Theil 1972). Zero means a perfect fit.

Using the RM and HI data we can estimate the preferred radial direction of magnetic field, e.g., inward or outward of the galactic center (cf. Krause \& Beck 1998). The kinematical properties of the spiral structure in NGC 4254 can provide information on the sense of the galaxy's rotation. As argued by

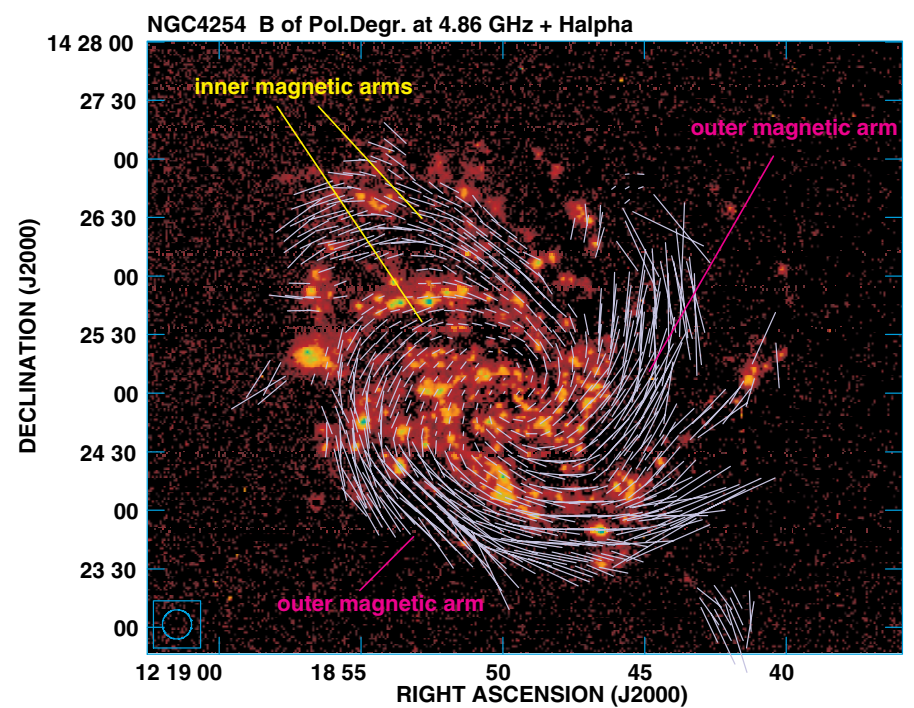

Fig. 4. Actual (Faraday-free) structure of magnetic field vectors in NGC 4254 obtained from the combined VLA and single-dish (Effelsberg) polarized data at $8.46 \mathrm{GHz}$ and $4.86 \mathrm{GHz}$. The lengths of vectors are proportional to the degree of polarization at $4.86 \mathrm{GHz}$. The regions with inner and outer magnetic arms are designated. Overlaid (in colors) is the $\mathrm{H} \alpha$ image (from Knapen et al. 2004).

Phookun et al. (1993), the spiral arms in NGC 4254 are generally of trailing type, although a small fraction of stars rotate in reverse. From the H I velocity field we know that the western part of the galaxy is in approaching motion, thus the southern part of the disk is the nearest side. As both the radial velocity and $R M$ have the same sign on both ends of the galaxy's major axis (in particular, magnetic fields are directed outwards from the observer in the western part), we conclude that the regular (coherent) field in NGC 4254 is oriented outwards from the disk center, which is contrary to most observed galaxies (Krause \& Beck 1998). The outwards directed field was observed to date only in the disk of M 51. Thus, in both galaxies, outwards fields could possibly arise from the external interaction with a companion.

With the distribution of $R M$ available at hand, we can reconstruct the intrinsic position angle of the magnetic field over the whole galaxy, free of Faraday rotation. We assume a simple quadratic relation of $R M$ to wavelength, which should be an appropriate approximation for the high frequency data used. The actual magnetic field structure thus corrected is presented in Fig. 4 with vector lengths proportional to the degree of polarization at $4.86 \mathrm{GHz}$. The magnetic structure is smooth and of spiral pattern, even within the galaxy inner $1^{\prime}$ region, where the optical $(\mathrm{H} \alpha)$ spiral structure is unclear.

The visible magnetic arms in Fig. 4 are mainly interlaced with optical $(\mathrm{H} \alpha)$ ones. However, we distinguish "inner" magnetic arms, which are displaced off the optical ones to their inner sides and the "outer arms", which are shifted off outwards, to the downstream side of a density wave. In the next section, we show that both kinds of magnetic arms differ in magnetic properties, which must have been due to some differences regarding their origin. In general, the pattern of magnetic arms in NGC 4254 apparently follow the structure of local optical features, which indicates a substantial influence of density waves on magnetic pitch angle (see Sects. 3.1 and 3.5). 


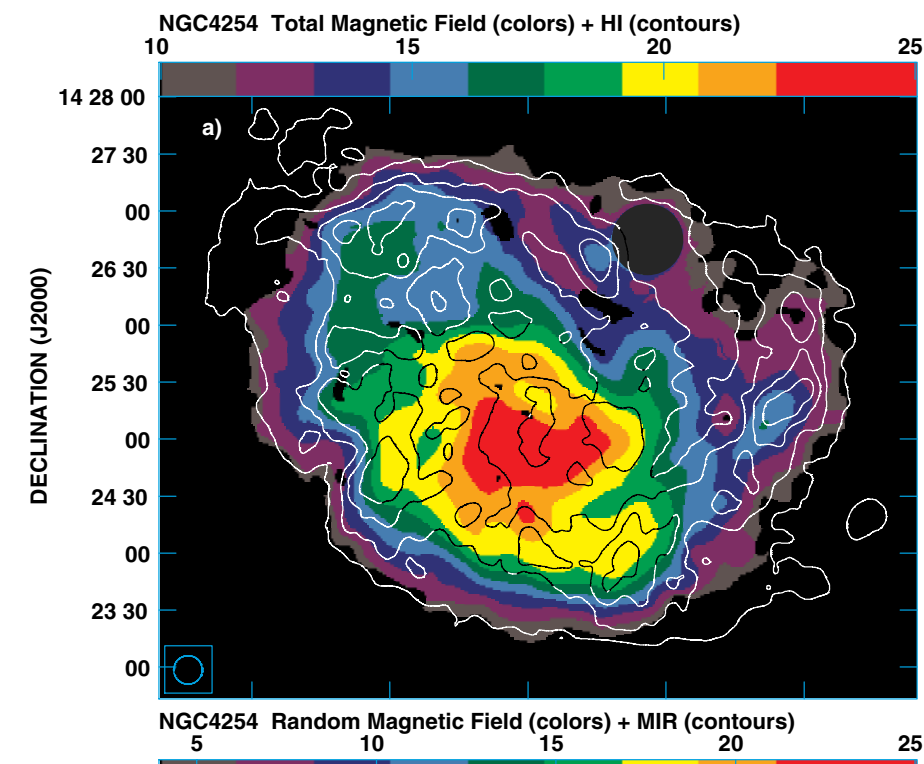

NGC4254 Regular Magnetic Field (colors) + Halpha (contours)
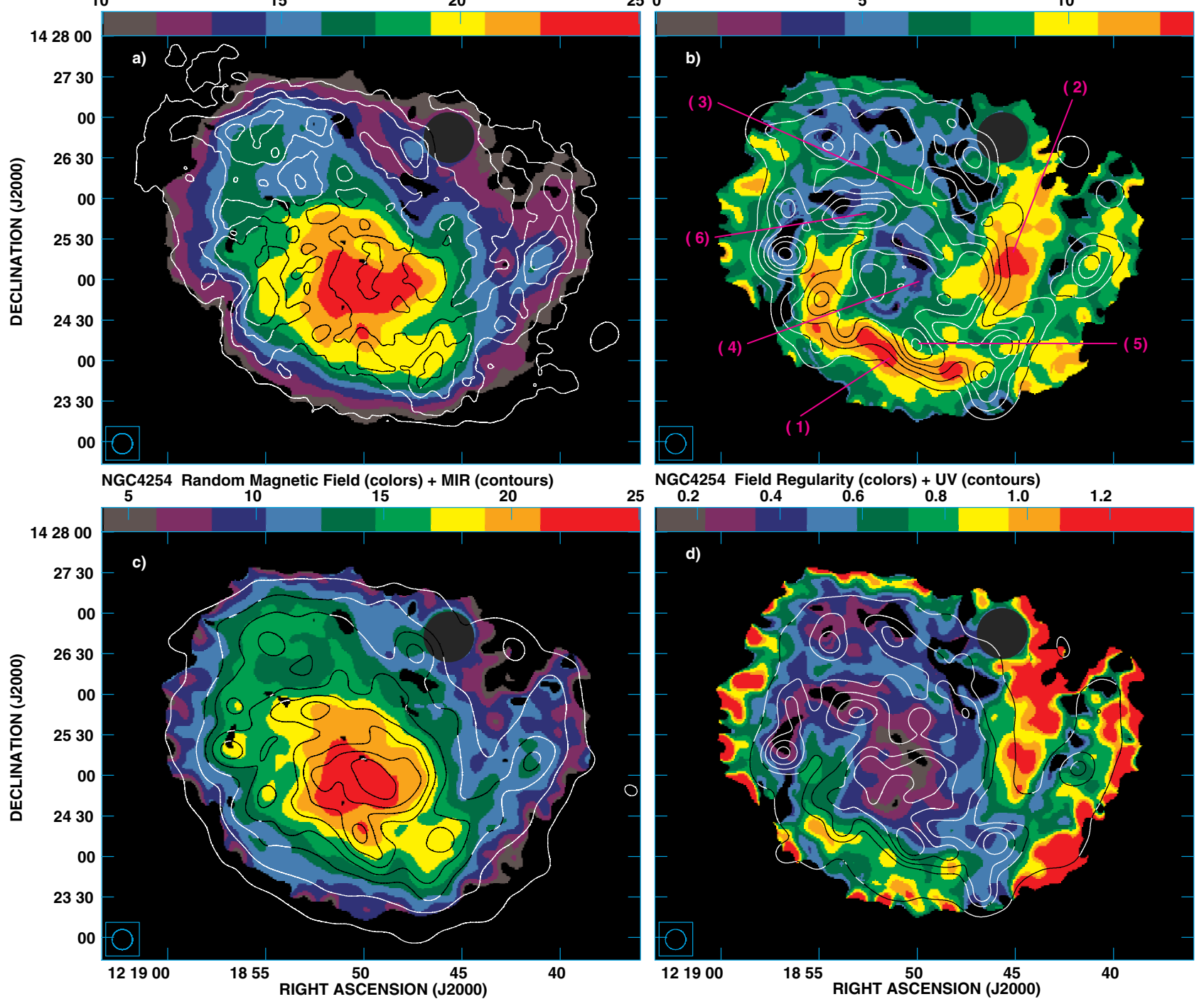

NGC4254 Field Regularity (colors) + UV (contours)

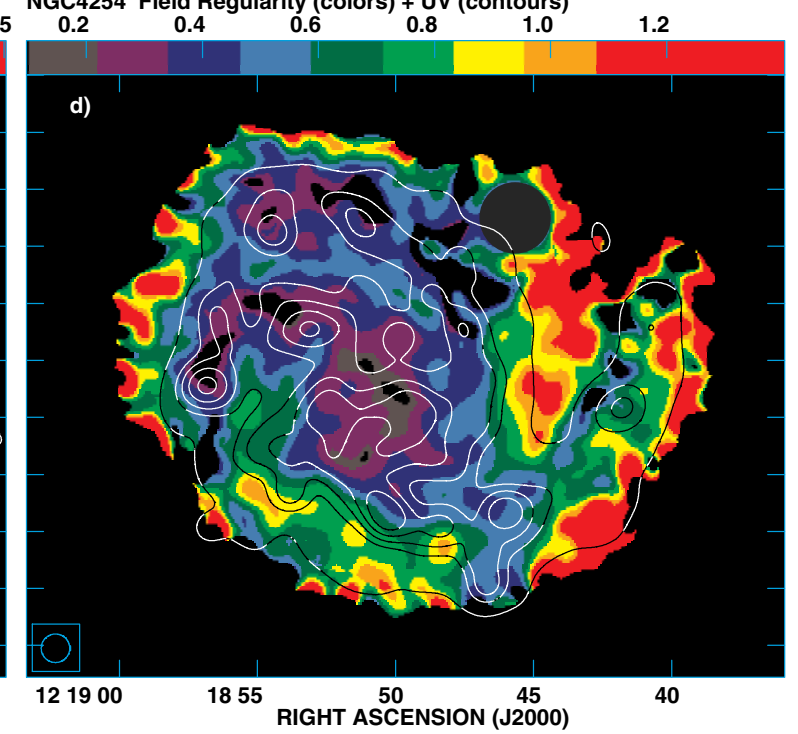

Fig. 5. Magnetic maps for NGC 4254 with a resolution of $15^{\prime \prime}$ (in colors): total a), regular b), random c) magnetic field strength, and field regularity d) with contours of $\mathrm{HI}, \mathrm{H} \alpha$, infrared $(24 \mu \mathrm{m})$, and UV emission, respectively. The method of the maps construction is given in Sect. 2.3.

\subsection{Magnetic maps}

Currently, magnetic properties of galaxies are most often presented using the field strength estimated in a small number of individual regions within a galaxy or as the average for the whole disk. In the case of complicated magnetic field structures as, e.g., in the Antennae system of interacting galaxies proper interpretation of polarimetric data requires a comparison of field strength in the regular and random components in many different regions over the entire system (Chyży \& Beck 2004). The magnetic field in the perturbed spiral NGC 4254 is quite complicated too, showing unusual polarization features and $R M$ pattern, different kind of magnetic arms in various parts of the galaxy, and thus requires a similar mode of analysis. We, therefore, propose here to present for the first time galactic magnetic field in a form of complete "magnetic maps" that show the distributions of strengths of intrinsic magnetic field components in all available regions in the galaxy disk, with the galaxy projection and Faraday rotation effects removed.

The strength of intrinsic regular component of magnetic field $B_{\text {reg }}$, corrected for galaxy inclination and varying angle between the line of sight and the field line, is derived from the observed radio polarized intensity. We assume that the regular magnetic field lies only in the galaxy plane, which permits derivation of the intrinsic (in the disk plane) magnetic field position angle from the derived sky-projected (but Faraday rotation free) polarization position angle (Sect. 2.1) and the galaxy orientation. Following Phookun et al. (1993) we adopt values of $42^{\circ}$ and $68^{\circ}$, respectively, for the galaxy inclination and the major axis position angle. The intrinsic (de-projected) total magnetic field strength $B_{\text {tot }}$ is calculated through a similar procedure using, in this case, the total nonthermal (synchrotron) intensity. The random magnetic field component $B_{\text {ran }}$ is derived by subtracting the reconstructed intrinsic regular field from the total field.

In our calculations, we apply the energy ratio $k=100$ of relativistic protons and electrons, and a $300 \mathrm{MeV}$ cutoff in the cosmic rays (CR) proton spectrum (e.g., Ehle \& Beck 1993; Chyży $\&$ Beck 2004). We assume the equipartition energy condition between CRs and magnetic fields and apply a constant nonthermal spectral index $\alpha_{\text {nth }}=1.0$ (cf. Paper I). The magnetic field is supposed to be located within the plane of the galaxy disk (without a vertical component) of about $1 \mathrm{kpc}$ unprojected thickness. The whole described procedure of magnetic maps' derivation 
Table 2. The intrinsic magnetic field strength of total $B_{\text {tot }}$ and regular $B_{\text {reg }}$ components as well as the field regularity in different regions of NGC 4254, denoted in Fig. 5 (top-right). The given uncertainties express random noise in the maps used. The errors, including possible systematic uncertainties due to applied assumptions, can reach $25 \%$ of the values obtained. The presented magnetic fields strengths are taken directly from magnetic maps described in Sect. 2.3.

\begin{tabular}{lrrr}
\hline \hline & $\begin{array}{r}B_{\text {tot }} \\
{[\mu \mathrm{G}]}\end{array}$ & $\begin{array}{r}B_{\text {reg }} \\
{[\mu \mathrm{G}]}\end{array}$ & $B_{\text {reg }} / B_{\text {ran }}$ \\
\hline Total & $16 \pm 1$ & $7 \pm 0.2$ & n.a. \\
1) Southern outer magnetic arm & $19.7 \pm 0.5$ & $12.6 \pm 0.3$ & $0.83 \pm 0.03$ \\
2) Western outer magnetic arm & $18.9 \pm 0.8$ & $12.3 \pm 0.4$ & $0.86 \pm 0.09$ \\
3) Northern inner magnetic arm & $16.9 \pm 0.2$ & $7.8 \pm 0.3$ & $0.52 \pm 0.02$ \\
4) Central part & $24.3 \pm 0.5$ & $4.8 \pm 0.7$ & $0.20 \pm 0.03$ \\
5) SW optical spiral arm & $22.2 \pm 0.3$ & $7.4 \pm 0.2$ & $0.35 \pm 0.02$ \\
6) NE optical spiral arm & $20.3 \pm 0.7$ & $6.5 \pm 0.8$ & $0.34 \pm 0.05$ \\
\hline
\end{tabular}

was implemented as a task in the AIPS ${ }^{4}$. The largest uncertainties in the obtained magnetic field strengths are most likely to be the systematic ones due to the assumed above values for the unknown parameters, rather than random errors due to uncertainties in observed total and polarized radio intensities (see below).

The first magnetic map of NGC 4254 presented in Fig. 5a shows the distribution of total magnetic field strength with contours of $\mathrm{HI}$ intensity for reference sake. In the central disk region the strength reaches $25 \mu \mathrm{G}$, while decreasing to about $10 \mu \mathrm{G}$ at the outer galaxy parts (see also Table 2). The strong localized signal in the disk northern periphery $\left(\mathrm{RA}=12^{\mathrm{h}} 18^{\mathrm{m}} 46^{\mathrm{s}}\right.$, Dec $\left.=14^{\circ} 26^{\prime} 45^{\prime \prime}\right)$ coming from a confusing background source is masked in this and following magnetic maps. The mean strength of total magnetic field over the whole galaxy (without the background source) is $16 \pm 1 \mu \mathrm{G}$, which is in agreement with the low-resolution estimation by Soida et al. (1996). This value is higher than typical ones (of about $10 \mu \mathrm{G}$ ) found in a large sample of galaxies (Beck et al. 1996) but closer to the mean field strength in the disks of the Antennae galaxies (about $20 \mu \mathrm{G}$, Chyży \& Beck 2004). As can be seen from Fig. 5a (and Table 2), the sites of star formation and effective production of CRs in optical spiral arms possess a strong magnetic field of about $16-22 \mu \mathrm{G}$. In the interarm regions, the total field weakens typically to $14-17 \mu \mathrm{G}$. We mention that in some analyses of galaxies of Hubble type similar to NGC 4254 a disk thickness of $0.5 \mathrm{kpc}$ is assumed. If, by way of comparison, this value is applied to NGC 4254, the corresponding total magnetic field in the optical arms rises to $19-25 \mu \mathrm{G}$ and to $30 \mu \mathrm{G}$ in the disk core.

The second magnetic map of intrinsic regular field is presented in Fig. $5 \mathrm{~b}$ with contours of $\mathrm{H} \alpha$ emission. The typical strength of regular field in the $\mathrm{N}$ and NE optical arms is about $5 \mu \mathrm{G}$. In the inner magnetic arm (region 3) it rises up to $7.5-8 \mu \mathrm{G}$. In the $\mathrm{SW}$ optical spiral arm, the regular field reaches $6-7 \mu \mathrm{G}$, while outside it, in the southern outer magnetic arm (in the strongly-polarized ridge, region 1) it is enhanced up to $13 \mu \mathrm{G}$. The large interarm region between $\mathrm{SW}$ and $\mathrm{N}$ optical arms maintains over a large area, a strong regular field of about $10 \mu \mathrm{G}$, stronger than in the northern inner magnetic arm. In the western outer magnetic arm (region 2), it reaches a value even as strong as the regular field in the southern polarized ridge: $12-13 \mu \mathrm{G}$.

The distribution of intrinsic regular field shows a global asymmetry in the SW-NE direction that was partly hidden due to

\footnotetext{
${ }^{4}$ Astronomical Imaging Processing System of the National Radio Astronomy Observatory.
}

projection effects in the distribution of polarized intensity, which shows just a strong N-S asymmetry (Fig. 1, Paper I). The strong regular field, manifested not only in the southern galactic outskirts, but also in the western outer magnetic arm (region 2) inside the galactic disk, must have profound consequences. This introduces a question of the origin of the field in both the regions, and their possible connection.

A map of the turbulent component of magnetic field (Fig. 5c) shows an excellent correlation with the dust emission (in contours) observed in mid-infrared (MIR) by Spitzer (see Paper I). This can indicate a strong production of turbulent field or a strong tangling (de Avillez \& Breitschwerdt 2005) in sites of star-forming regions revealed without extinction by the MIR emission. Surprisingly, in the place of strongly-increased regular field in the southern outer magnetic arm no such enhancement is visible in the random field. We model this behavior of different magnetic field components in Sect. 3.4.

The ratio of derived (projection free) regular to random magnetic field components - the field regularity $B_{\text {reg }} / B_{\text {ran }}$ - is a useful measure of the net production of regular field independent of magnetic field strength, as well as a sensitive tracer of local processes in magnetized plasma. We present the distribution of field regularity as yet another "magnetic map" in Fig. 5d, together with the contours of UV emission associated with population of young stars and the regions of most turbulent ISM. In fact, along the three main optical spiral arms and in the galaxy center the field regularity is low (0.2-0.5). Both the magnetic outer arms (region 1 and 2) have an exceptionally high-field regularity of about 0.8 , in contrast to a typical value of 0.5 in other interarm regions (including region 3). As it could be expected, the regularity is also larger when going outside turbulent regions into the galaxy outskirts. Hence, such a highly-regular magnetic field in the galactic halo is likely to enter the Virgo Cluster's medium.

With the magnetic maps at hand, we also looked at where the magnetic energy dominates the total energy in the thermal gas component. We derived the distribution of the latter quantity from the emission measure estimated from radio thermal emission (Paper I). The magnetic energy exceeds the thermal one across almost the entire galaxy (except for just a few regions of very intensive star formation). Moreover, it exceeds the turbulent gas energy (estimated from the dispersion in the H I velocity field, Paper I) in the southern polarized ridge, in the large western interarm region, and in the whole polarized galactic outskirts visible at $4.86 \mathrm{GHz}$ and $1.4 \mathrm{GHz}$. Thus, in those regions the magnetic field can be even dynamically important (as in NGC 6946; Beck 2007).

In the above estimations of the magnetic field strength, we assumed a constant synchrotron spectral index $\alpha_{\text {nth }}=1.0$ across the galaxy, while it may attain smaller values in star-forming regions and larger ones in the galaxy's outskirts. Such variations, if known, could easily be incorporated in the procedures calculating the magnetic maps. Fortunately, the strength of the magnetic field only weakly depends on $\alpha_{\text {nth }}$. For example, we performed an additional separation of thermal and nonthermal radio emission assuming $\alpha_{\mathrm{nth}}=0.8$, which is more suitable for spiral arms. Then, we derived the magnetic maps with the new $\alpha_{\text {nth }}$ and compared them with the previous ones. In spiral arms and in the galactic centre the differences are up to about $2 \mu \mathrm{G}$. In particular, in the optically-bright region in the NE spiral arm at $\mathrm{RA}=12^{\mathrm{h}} 18^{\mathrm{m}} 53^{\mathrm{s}}$, Dec $=14^{\circ} 25^{\prime} 51^{\prime \prime}$ the total field strength is smaller by $1.3 \mu \mathrm{G}$, the regular one by $0.58 \mu \mathrm{G}$, and the random one by $1.4 \mu \mathrm{G}$, giving the field regularity of 0.22 , instead of 0.30 before. Due to energy losses of the CR electrons, the field strength can be underestimated in the outer part of the galaxy 


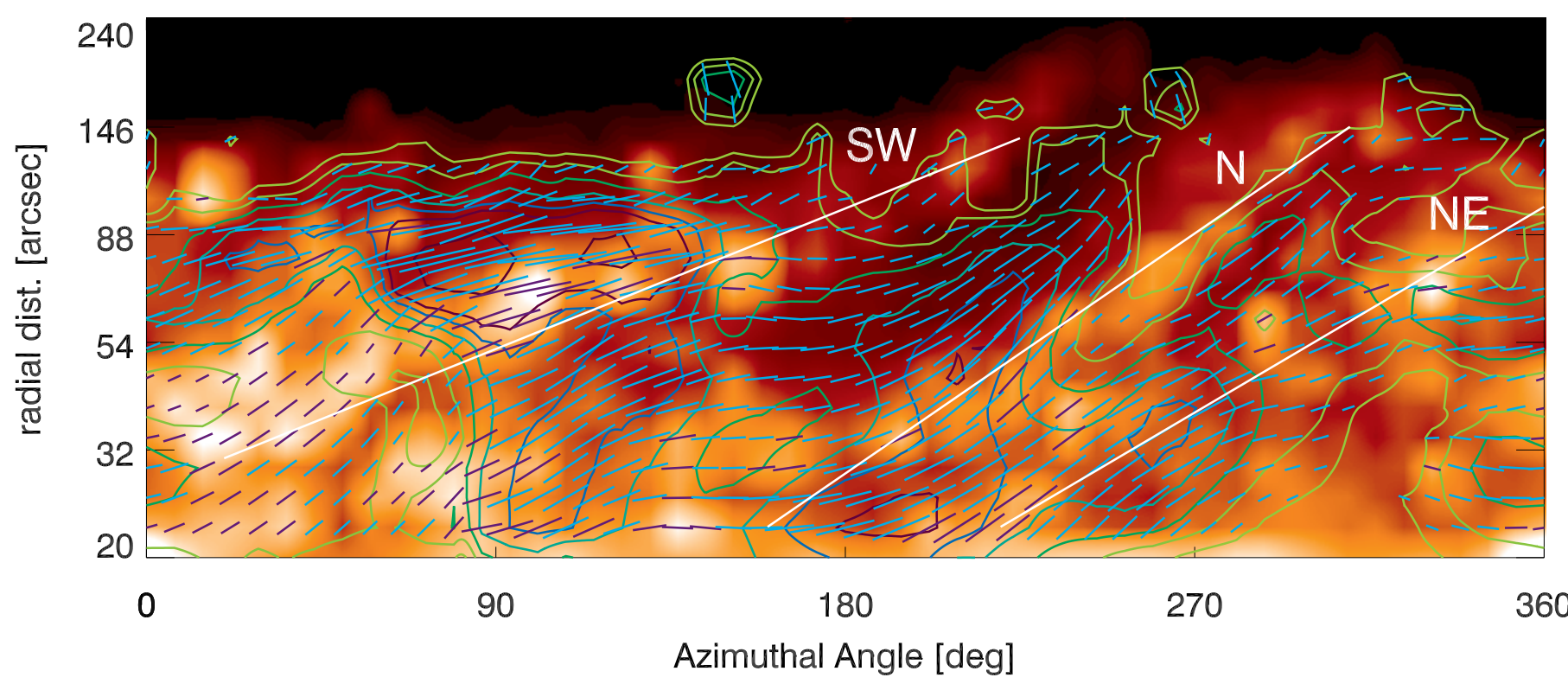

Fig. 6. A phase diagram of the regular component of magnetic fields in dependence of natural logarithm of distance and galactocentric azimuthal angle, as measured from the northern tip of the major axis counterclockwise. The vectors are proportional to the polarized intensity at $4.86 \mathrm{GHz}$, which is also presented in contours. In the frame of this representation, a logarithmic spiral arm would be a straight line inclined by the pitch angle. The galactic spiral structure is shown by the distribution of UV emission (in color) with additionally marked (the straight lines) approximate orientations of the main three optical spiral arms. All the presented values were averaged in sectors of $7^{\circ}$ width in the azimuth.

due to a higher ratio $k$ of proton to electron energy. We estimated that effect by setting $k$ to 200 . Differences to the original maps are in this case up to about $3 \mu \mathrm{G}$. In the tip of western outer magnetic arm at RA $=12^{\mathrm{h}} 18^{\mathrm{m}} 44^{\mathrm{s}}$, Dec $=14^{\circ} 26^{\prime} 21^{\prime \prime}$ the total field is stronger now by $2.4 \mu \mathrm{G}$, the regular one by $2.1 \mu \mathrm{G}$, and the random one by $0.6 \mu \mathrm{G}$. The field regularity rose from 1.25 to 1.41. Such variations of parameters across NGC 4254, as discussed above, would not change conclusions of this paper.

\section{Discussion}

The intrinsic magnetic field vectors in NGC 4254 seem to follow the perturbed optical spiral pattern (Fig. 4). This suggests that apart from the dynamo action, an additional influence of gas flows is involved. The $R M$ distribution also reveals a perturbed axisymmetric dynamo mode or a mixture of two modes and an unusual outward directed orientation of the magnetic field (Sect. 2.2). According to the magnetic maps, the total magnetic field is stronger in NGC 4254 than in a typical field galaxy (Fig. 5, Table 2). Such higher magnetic activity can be associated with the high SFR manifested by this galaxy (Paper I). However, several magnetic arms with strong regular fields located on the downstream and upstream sides of local density waves cannot be readily accounted for and different physical processes could be involved. The strong regular field (of about $12 \mu \mathrm{G}$ ) revealed not only in the southern galactic outskirts, but also in the western outer magnetic arm, well inside the galactic disk, suggests a possibility that both the magnetic structures could have arisen from the same phenomena. To address all these problems, below we analyze the correspondence of the orientation of the magnetic field with the optical spiral arms and the connection of the regular field with star-forming regions. Subsequently, we model depolarization and magnetic field components, according to different physical phenomena.

\subsection{Orientations of magnetic field vectors}

We can explore the relation between the magnetic and perturbed optical spiral arm pattern in NGC 4254 by investigating the orientation of magnetic field vectors with respect to the main optical spiral arms. We constructed a phase diagram of orientations of such vectors, $R M$-corrected and de-projected, as a function of radius $R$ (in natural logarithm) and galactocentric azimuthal angle.

Throughout large galaxy domains the magnetic field vectors show a similar pitch angle (Fig. 6). In the inner disk part $\left(R<50^{\prime \prime}\right)$ two magnetic patterns are observed. The dominant one has a coherent large pitch angle $\left(>25^{\circ}\right)$, regardless of the chaotic pattern of $\mathrm{H} \alpha$ and UV emitting gas. This pattern is broken in two regions (around the azimuthal angle of $150^{\circ}$ and $340^{\circ}$ ) with almost azimuthal magnetic field. Small pitch angles could be associated with the central bar-like structure seen in high-resolution (7!'5) radio maps (Paper I). Indeed, the position angle of the bar visible in high-resolution radio observations (see Fig. 3 in Paper I) is $55^{\circ}$ giving the azimuthal angles of the bar ends in the regions of low values of the magnetic pitch angle. A similar configuration of magnetic field (with azimuthal field close to the bar ends) is visible in the strongly-barred galaxy NGC 1365 (Moss et al. 2007). However, the performed MHD simulation was unable to reproduce this magnetic pattern in detail (the mean difference between observed and simulated pitch angles is $30^{\circ}$ ). In order to fully explain the magnetic field within the inner disk of NGC 4254, an advanced MHD simulation of a weakly barred galaxy is needed.

In the southern polarized ridge (radius $60^{\prime \prime}-100^{\prime \prime}$ and azimuth $45^{\circ}-150^{\circ}$ ), the regular magnetic field changes its pitch angle and the polarized emission is shifted outside the local UV-maxima. The magnetic vectors seem to be aligned with the optical (and UV) SW spiral arm, which has an oscillating pattern around the pitch angle of about $20^{\circ}$ (marked in Fig. 6). 
At larger azimuth $\left(>180^{\circ}\right)$, the $\mathrm{N}$ and $\mathrm{NE}$ optical spiral arms have different average pitch angle than the $\mathrm{SW}$ arm $\left(30^{\circ}\right.$ and $25^{\circ}$, respectively), providing further evidence for the perturbed spiral pattern of NGC 4254. The N optical arm is heavily curved at large distances $\left(r>30^{\prime \prime}\right)$ and its pitch angle is reduced to zero. The $\boldsymbol{B}$-vectors trace the orientation of both the optical arms and deviate from them only slightly (up to $20^{\circ}$ ), even at the tip of the $\mathrm{N}$ arm. The polarized maxima are shifted from the optical spiral arms, especially on the downstream side of the $\mathrm{N}$ arm. In this region (between $\mathrm{SW}$ and $\mathrm{N}$ arms), the magnetic pitch angle reaches a locally large value of $50^{\circ}$. We showed in Sect. 2.3 that in this part of the galaxy the energy density of the ISM can be dominated by the magnetic component, thus probably allowing the magnetic field to form its own configuration.

The following of the perturbed optical spiral arms by the vectors of the regular magnetic field suggests a physical association of both patterns. Similar correlations has been observed for some grand-design spirals, e.g., in M 51 (Fletcher et al. 2004b; Patrikeev et al. 2006). The alignment of $\boldsymbol{B}$-vectors with CO pitch angles in M 51 was suggested to results from the shock compression along the upstream side of spiral density waves. The northern part of NGC 4254 is much more flocculent than the spiral structure in M51, which implies that the suggested interaction must still hold for weak density waves and for a perturbed disk. However, the downstream magnetic arms in NGC 4254 cannot be explained by this process.

Locally, large magnetic pitch angle correlated with orientation of spiral arms in NGC 4254 is not attainable in the classical MHD dynamo model (Elstner et al. 2000). Relatively-large, magnetic pitch angles (up to $40^{\circ}$ ) could still be obtained in MHD dynamo by allowing an increase in the correlation time of interstellar turbulence (Rohde \& Elstner 1997; Rohde et al. 1999). However, the magnetic pitch angle was found in this case not to depend on the pitch angle of the gaseous arms. Furthermore, this approach was criticized by Shukurov (2005), who argued for shorter correlation time in spiral arms, contrary to the authors above. MHD simulations by Elstner et al. (2000), which include an enhancement of turbulent diffusion in spiral arms and density wave-alike gas velocity patterns, produced the influence of gas flows on dynamo-generated magnetic fields. However, the generated magnetic pitch angle in the interarm regions are smaller than within spiral arms. This is not actually observed in NGC 4254: in the large interarm region between SW and N optical arms, the pitch angle is the largest in the whole galaxy (up to $50^{\circ}$ ). Further investigation by MHD modeling to show whether gas flows or high turbulent diffusion are able to maintain such a large pitch angle in NGC 4254 is highly needed.

Addressing the problem of observed phase shifts between the magnetic and optical arms in NGC 4254, we mention the work of Shukurov (1998), who suggested that a similar shift observed in NGC 6946 can result from a certain time lag between an enhancement in turbulence within density waves and the response in dynamo coefficients. This cannot account for the outer magnetic arms in NGC 4254; the lag in these cases would be negative. There is another possibility given by Shukurov explaining magnetic arms in NGC 6946 as a suppression of dynamo efficiency in the gaseous arms by an enhanced turbulent magnetic diffusivity. However, such a shift becomes smaller along the SW arm and disappears in the western part of the arm (azimuth $>140^{\circ}$ ), contrary to the expectations, since a larger distance from the galaxy center should result in a smaller dynamo number and a higher shift (as observed, e.g., in M 51, Fletcher et al. 2004b). Hence, the magnetic arms of NGC 4254 and the large magnetic pitch angle indicate a strong influence of gaseous flows on the dynamo-induced magnetic fields, and possibly some external cause, such as compression or shear, at work (see below).

\subsection{Magnetic field regulation by SFR}

Magnetic maps enable analyzing the correlation between the strength of various components of the magnetic field and the star formation activity over different regions of NGC 4254. In Fig. 7a, we present such a relation as based on $24 \mu \mathrm{m}$ SFR (cf. Paper I) and the magnetic map of total field strength (Fig. 5a). The observed relation for 277 beam independent (15" spaced) regions over the galaxy is a single power-law throughout almost three orders of magnitude in SFR. The relation is tight, with the Pearson correlation coefficient of 0.93 , and has the form fitted by the bisector method:

$\log B_{\text {tot }}=0.18( \pm 0.01) \log \mathrm{SFR}+1.56( \pm 0.01)$.

This relation is also another example of the well-known radio/IR correlation discussed in Paper I and reflects a process of scaling the production of magnetic field and CRs with star formation activity.

We also separated regions of $\mathrm{S}, \mathrm{W}$, and $\mathrm{N}$ magnetic arms and marked them all in Fig. 7. We repeated the fitting procedures for them. The resulting slopes $a$, offsets $b$, correlation coefficients $r$, and number of used regions $\mathrm{N}$ are given in Table 3 . The regions of outer magnetic maps have relatively-high total field strength as compared to the inner $\mathrm{N}$ magnetic arm.

The intensive star formation is generally considered to produce an increased level of turbulence and to influence the regular magnetic field. However, this process has not been quantitatively investigated to date. Now, for the first time, this can be done with the constructed magnetic maps for NGC 4254. The relation between SFR and the regular field's strength scaled by the random field - i.e., the field regularity - is presented in Fig. 7b for 234 independent galaxy regions. The relation is well described by a power-law that we estimated by the bisector method, which yields:

$\log \frac{B_{\text {reg }}}{B_{\text {ran }}}=-0.32( \pm 0.01) \log$ SFR $-0.90( \pm 0.03)$.

It shows that contrary to the total magnetic field strength, the field regularity strongly decreases with the star-formation level: the observed anticorrelation is -0.71 . Several processes can explain the observed trend: 1) field tangling by some processes related to the star formation; 2) production of turbulent fields proportional to SFR (by a turbulent dynamo or any other turbulent field amplification mechanisms); and 3) production of regular field anti-proportional to SFR. The latter can be achieved with the mean-field dynamo effects anti-proportional to SFR, e.g., by the correlation length of turbulence anti-proportional to SFR, as proposed by Rohde et al. (1999), or by the turbulent diffusivity enhanced by shear, as proposed by Moss et al. (2001) and Moss et al. (2007), or by the suppression of mean-field dynamo action in spiral arms (Shukurov 2005). As all the above concepts give different predictions for scaling of regular and random magnetic field with SFR, we present separately the strength of both the magnetic components versus SFR in Figs. 7c, $d$ and the fitted power-laws in Table 3. Strikingly enough, there is a strong correlation of random field $B_{\text {ran }}$ with SFR $(r=0.91$ for all regions) and no such correlation for regular field $(r=0.08)$. This suggests that the effective production of random magnetic field in the star-forming regions (i.e., the second possibly underlying process from those listed above) is the principal cause of the strong anticorrelation of field regularity and SFR (Fig. 7b). 

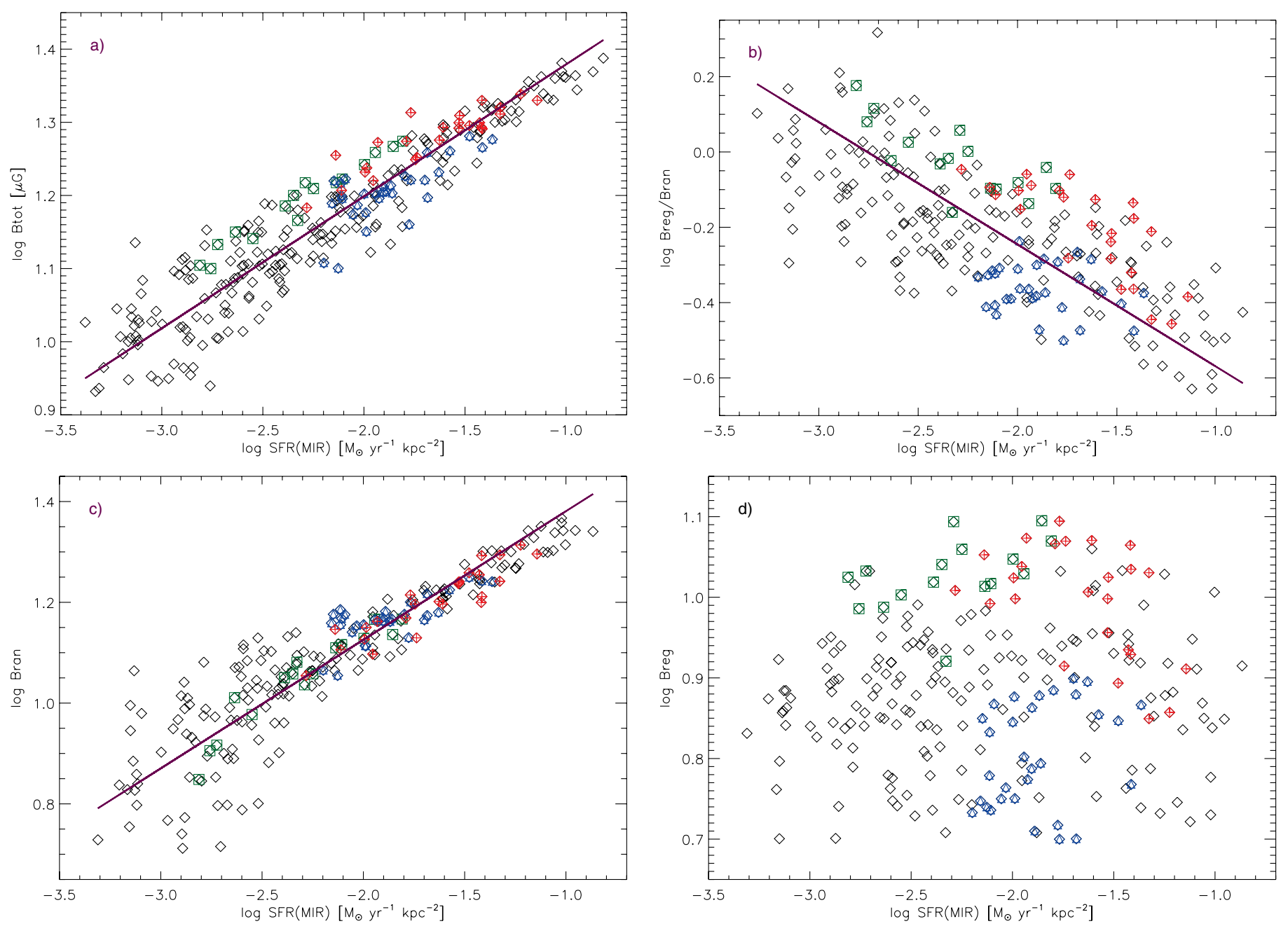

Fig. 7. Total magnetic field strength a), field regularity b), random c), and regular d) magnetic field strengths versus IR-based SFR within NGC 4254. From among all regions (marked by diamond sign) magnetic arms are denoted separately: southern outer magnetic arm by crosses; western outer magnetic arm by rectangles; and northern inner magnetic arm by triangles. The lines fitted by bisector method to all regions are drawn (except d-panel, see Sect. 3.2).

However, the $B_{\text {reg }}-$ SFR relation shows a two-way behavior (Fig. 7d) for stronger regular fields with an upper envelope slightly raising for weak SFR, and declining for the large SFR. The dividing value is $\mathrm{SFR} \approx 0.016 M_{\odot} \mathrm{yr}^{-1} \mathrm{kpc}^{-2}$, which corresponds to $B_{\mathrm{ran}} \approx 14 \mu \mathrm{G}$ and to the maximum of $B_{\mathrm{reg}}$ of about $12.5 \mu \mathrm{G}$. This two-way trend is apparent in the data in Table 3 as positive and negative correlation coefficients for western and southern magnetic arms, respectively, and an insignificant correlation when all galactic regions are taken together. Hence, the production of regular field in NGC 4254 could have been suppressed in the most vivid star-forming regions (process 3 ) or the regular field could have been disrupted by an efficient field tangling (process 1 above). Further analyses of magnetic structures in other galaxies and advanced simulations of MHD dynamo involving various SFRs are needed to fully account for these relations.

The regions of $\mathrm{S}$ and $\mathrm{W}$ outer magnetic arms reveal a distinct behavior from the other galactical regions. Their regular field strength reaches the highest attainable values, which places them on the top of $B_{\text {reg }}-$ SFR relation (Fig. 7d). In contrast, their random field is average, situating them close to the fitted powerlaw for all the galactic regions (Fig. 7c). Thus, the stronger total field for the outer magnetic maps mentioned above is due to the stronger regular field and not the random one.
This behavior is well rendered by the field regularity data. On the field regularity-SFR diagram (Fig. 7b) the outer magnetic arms approach large values at the upper envelope of the relation. In contrast, the regions of the inner $N$ magnetic arm are distributed mostly below the average trend determined for all the galaxy regions (the fitted line in Fig. 7b). This can indicate that the regular field in the outer magnetic arms is probably not fully controlled by the star formation, as for instance in the southern outer magnetic arm, which shows a high-field regularity and does not avoid the high-SFR regions. Apparently, some additional process to regularize magnetic fields or a more efficient production of regular field component must be at work within the outer magnetic arms (which we explore in Sect. 3.4).

We also investigate how the applied assumption of constant nonthermal spectral index $\alpha_{\text {nth }}$ in derivation of magnetic maps in Sect. 2.3 could influence the discussed above relations. We use from Sect. 2.3 a rough estimation of possible changes of the total magnetic field in the NE spiral arm and in the tip of the western outer magnetic arm. We predict a possible slight decrease of the slope in $B_{\text {tot }}-$ SFR relation which would, however, give a powerlaw fit within the current spread of points in Fig. 7a. Similar considerations predict a possible small decrease of the slope of $B_{\text {ran }}$-SFR relation (Fig. 7c) and an increase of the anticorrelation in the field regularity-SFR relation (Fig. 7b), again within 
Table 3. The fitted relations between total $B_{\text {tot }}$, random $B_{\text {ran }}$, and regular $B_{\text {reg }}$ magnetic field strength, and the field regularity $B_{\text {reg }} / B_{\text {ran }}$, versus star-formation rate SFR for all regions of NGC 4254 and for different magnetic arms.

\begin{tabular}{lllll}
\hline \hline Regions & $a$ & $b$ & $r$ & $N$ \\
\hline all & \multicolumn{1}{l}{$\log B_{\text {tot }}=a \log \mathrm{SFR}+b$} \\
$\mathrm{~S}$ arm & $0.18 \pm 0.01$ & $1.56 \pm 0.01$ & +0.93 & 277 \\
$\mathrm{~W}$ arm & $0.13 \pm 0.01$ & $1.50 \pm 0.02$ & +0.89 & 25 \\
$\mathrm{~N}$ arm & $0.19 \pm 0.01$ & $1.59 \pm 0.02$ & +0.98 & 16 \\
\hline \multicolumn{5}{c}{$\log B_{\text {ran }}=a \log \mathrm{SFR}+b$} \\
all & $0.26 \pm 0.01$ & $1.64 \pm 0.02$ & 0.91 & 234 \\
$\mathrm{~S}$ arm & $0.22 \pm 0.02$ & $1.57 \pm 0.03$ & 0.91 & 25 \\
$\mathrm{~W}$ arm & $0.30 \pm 0.03$ & $1.73 \pm 0.06$ & 0.95 & 16 \\
$\mathrm{~N}$ arm & $0.20 \pm 0.03$ & $1.55 \pm 0.05$ & 0.72 & 31 \\
\hline \multicolumn{5}{c}{$\log B_{\text {reg }}=a \log \mathrm{SFR}+b$} \\
all & two-way trend & 0.08 & 234 \\
$\mathrm{~S}$ arm & $-0.28 \pm 0.04$ & $0.52 \pm 0.07$ & -0.49 & 25 \\
$\mathrm{~W}$ arm & $0.17 \pm 0.07$ & $1.43 \pm 0.15$ & 0.46 & 16 \\
$\mathrm{~N}$ arm & $0.47 \pm 0.16$ & $1.69 \pm 0.05$ & 0.28 & 31 \\
\hline \multicolumn{5}{c}{$\log \frac{B_{\text {reg }}}{B_{\text {ran }}}=a \log \mathrm{SFR}+b$} \\
all & $-0.32 \pm 0.01$ & $-0.90 \pm 0.03$ & -0.71 & 234 \\
$\mathrm{~S}$ arm & $-0.42 \pm 0.05$ & $-0.90 \pm 0.08$ & -0.78 & 25 \\
$\mathrm{~W}$ arm & $-0.30 \pm 0.04$ & $-0.71 \pm 0.11$ & -0.75 & 16 \\
$\mathrm{~N}$ arm & $-0.56 \pm 0.21$ & $-1.41 \pm 0.39$ & -0.21 & 31 \\
\hline
\end{tabular}

the spread of the data points. The variations of $\alpha_{\text {nth }}$ across the disk would induce an increase of regular field in galaxy outskirts and a decrease in star-forming regions, which we suspect could lead to a slight predominance of anticorrelation in the $B_{\text {reg }}-$ SFR relation (Fig. 7d).

\subsection{Modeling depolarization}

To establish the origin of magnetic field in NGC 4254, and the processes that underlie the observed regularity of magnetic field, it is necessary to understand the depolarization processes that influence the observed properties of polarized emission. In this section, we model various frequency-dependent depolarization effects in NGC 4254 by constructing distributions (maps) of expected depolarization and comparing them with observed distribution (Fig. 2b).

The line-of-sight component of coherent magnetic field embedded within the synchrotron emitting region and mixed with ionized gas can cause the differential Faraday rotation (Burn 1966). To obtain the modeled values of the depolarization in NGC 4254, we use the formula (Sokoloff et al. 1998):

$D P_{\mathrm{dFR}}=\left|\frac{\lambda_{2}^{2}}{\lambda_{1}^{2}} \frac{\sin \left(2 R M \lambda_{1}^{2}\right)}{\sin \left(2 R M \lambda_{2}^{2}\right)}\right|$

where $\lambda_{1}=0.062 \mathrm{~m}, \lambda_{2}=0.035 \mathrm{~m}$, and the rotation measure $R M$ values we take from the constructed $R M$ map (Fig. 2a).

The modeled depolarization, due to this effect, and observed depolarization are presented in Table 4 for some characteristic places within NGC 4254. Uncertainties of depolarization values estimated from their standard deviations in the measured regions are also given in Table 4. Throughout almost the entire galaxy the calculated depolarization is weak, $D P_{\mathrm{dFR}}$ values are close to about 0.95 . A larger modeled depolarization (with large statistical variations) appears in the central disk region, where $D P_{\mathrm{dFR}} \approx 0.7 \pm 0.3$, and in the regions of $R M$ jumps, where $D P_{\mathrm{dFR}}<0.2$, and where observed $D P$ are also similarly low. However, variations of depolarization are large in those regions.
The differential Faraday rotation does not account for the observed depolarization in the most NE disk portion (around 0.7, Fig. 2b), as it typically gives values around 0.9 .

As the second process of depolarization, we consider the internal Faraday dispersion produced by a turbulent magnetic field and thermal gas within the emitting volume (Burn 1966; Sokoloff et al. 1998). The fractional depolarization resulting from this process is given by:

$$
D P_{\text {disp }}=\frac{\lambda_{2}^{4}}{\lambda_{1}^{4}} \frac{1-\exp \left(-2 \sigma_{R M}^{2} \lambda_{1}^{4}\right)}{1-\exp \left(-2 \sigma_{R M}^{2} \lambda_{2}^{4}\right)} .
$$

The dispersion of rotation measure $\sigma_{R M}$ can be described using the "random walk" approach:

$\sigma_{R M}=0.812 B_{r} n_{\mathrm{c}} d \sqrt{N}$

where $B_{r}$ is the component of turbulent magnetic field in the direction of the observer, $d$ is the turbulent cell/cloud size (correlation length) of the random magnetic field, $n_{\mathrm{c}}$ is the electron density within the cloud, and $N$ is the cloud number along the line of sight. If the ionized diffuse gas has an extent $L$ toward the observer, and has a filling factor $f$, then its mean electron density $\langle n\rangle=f n_{\mathrm{c}}$ and $N=L f d^{-1}$. Similar results (with $\sigma_{R M}$ larger by up to two in Eq. (6)) are obtained using different approach (Beck et al. 2003).

The magnetic maps of random magnetic field strength allow us to derive a map of its line-of-sight component $B_{r}=B_{\operatorname{ran}} / \sqrt{3}$. Taking typical values of parameters: $d=50 \mathrm{pc}, L=1 \mathrm{kpc}$, and $f=0.5$ (cf. Beck 2007), we can adjust the only free parameter - the cloud density $n_{\mathrm{c}}-$ to get the best correspondence of the modeled $D P_{\text {disp }}$ with the actual map of observed depolarization within the spiral arms, where the Faraday dispersion is expected to be most pronounced. We find the best value $n_{\mathrm{c}}=0.08 \mathrm{~cm}^{-3}$ which yields $\langle n\rangle=0.04 \mathrm{~cm}^{-3}$, close to typical values used (e.g. $\langle n\rangle=0.03 \mathrm{~cm}^{-3}$ for NGC 6946; Beck 2007). Within these numbers Faraday dispersion can almost fully explain the actual low values of depolarization in the center of NGC 4254 giving at the same time good correspondence within the magnetic arms (Table 4). In the most NE part of the galaxy with rather weak thermal emission the obtained Faraday dispersion $\left(D P_{\text {disp }}>0.95\right)$ cannot account for the observed values (about 0.7).

We can also obtain the mean electron density from the emission measure $E M$ of diffuse thermal gas: $\langle n\rangle=\sqrt{f\left\langle n^{2}\right\rangle}=$ $\sqrt{f E M L^{-1}}$ which yields

$\sigma_{R M}=0.812 B_{r} \sqrt{E M d}$,

independent of the filling factor. The emission measure $E M$ can be estimated from our map of radio thermal emission of NGC 4254 at $8.46 \mathrm{GHz}$. Following Walterbos (2000), we assume that in NGC 4254, as in the Galaxy and in M 31, about $20 \%$ of the total ionized gas is diffuse, and that only this ISM phase contributes to $R M$ and to dispersion of $R M$. To make the modeled depolarization map consistent with observations, we must assume a correlation size $d$ in Eq. (7) of about 0.5 pc. However, this value is much smaller than applied above in Eq. (6), and which is usually assumed for other galaxies (see e.g., Fletcher et al. 2004a; Beck 2007). For regions of high EM $\left(>1000 \mathrm{pc} \mathrm{cm}^{-3}\right)$ it also evokes unreasonable small filling factor of the diffuse gas $f$, below 0.04. Either the used assumption in Eq. (7) of $20 \%$ diffuse gas is wrong, which would mean that NGC 4254 is a quite different galaxy from the Milky Way and M31 due to, e.g., a 
Table 4. Observed and modeled depolarization of synchrotron emission in several regions of NGC 4254 due to differential Faraday rotation, Faraday dispersion, and gradients in $R M$. The regions are marked in Fig. $5 \mathrm{~b}$.

\begin{tabular}{lrrrr}
\hline \hline & Obs. $D P(3.6 / 6.3)$ & Diff. FR & Far. Disp. & Grad. $R M$ \\
\hline 1) Southern outer magnetic arm & $1.08 \pm 0.02$ & $0.99 \pm 0.01$ & $0.99 \pm 0.01$ & $0.99 \pm 0.01$ \\
2) Western outer magnetic arm & $1.14 \pm 0.14$ & $0.97 \pm 0.02$ & $1.00 \pm 0.01$ & $0.98 \pm 0.01$ \\
3) Northern inner magnetic arm & $1.04 \pm 0.17$ & $0.99 \pm 0.01$ & $1.00 \pm 0.01$ & $0.98 \pm 0.01$ \\
4) Central part & $0.57 \pm 0.18$ & $0.66 \pm 0.28$ & $0.70 \pm 0.04$ & $0.91 \pm 0.11$ \\
5) SW optical spiral arm & $0.89 \pm 0.04$ & $0.99 \pm 0.01$ & $0.86 \pm 0.06$ & $0.99 \pm 0.01$ \\
6) NE optical spiral arm & $0.72 \pm 0.15$ & $0.91 \pm 0.07$ & $0.89 \pm 0.15$ & $0.94 \pm 0.04$ \\
\hline
\end{tabular}

much higher SFR, or our depolarization model is insufficient. An independent way to determine locally the amount of the diffused ionized emission in NGC 4254 is needed to solve this puzzle. Hence, fully understanding Faraday depolarization effects (as modeled by Eqs. (6) and (7)) seems essential in building a consistent model of the ISM in galaxies.

The third process, we will explore is depolarization $D P_{g F R}$ by gradients in the $R M$ across the observed synthesized beam of $\theta$ width, which occurs in a foreground screen (Sokoloff et al. 1998). We consider linear $R M$ gradients that are resolved with the observed beam. If the $R M$ has unresolved fine structure (within the beam size) then depolarization could be larger. Hence, in our approach the calculated $D P_{g F R}$ values represent an upper limit of depolarization. We constructed a relevant depolarization model map for NGC 4254 using AIPS task BDEPO, which use the following approximation:

$D P_{g F R}=\exp \left[\frac{-\left(g_{R M} \theta\right)^{2}\left(\lambda_{1}^{4}-\lambda_{2}^{4}\right)}{2 \ln 2}\right]$

where $g_{R M}$ is the gradient in $R M$. We estimate this gradient by applying the Sobol operator to the observed $R M$ map with the help of the AIPS task NINER.

The modeled $D P_{g F R}$ values outside the core of NGC 4254 are typically larger than 0.95 (Table 4). Relatively low values of $0.75-0.85$ are predicted for the NE outskirts of the galaxy, where observed depolarization is at a similar level and which could not be accounted for by other depolarization effects. As expected, gradients in $R M$ cause a large amount of depolarization in the $R M$ jump regions, where $D P_{g R M}$ goes down to $0.2-0.4$.

In summary, various depolarization processes dominate in different regions of NGC 4254, which explain the conclusion of Sect. 2.1, where we found no global correlation of depolarization with $R M$ or thermal emission. The depolarization observed in the central part of the galaxy is well accounted for by Faraday dispersion together with differential Faraday rotation (Table 4), while the large depolarization observed around $R M$ jumps can be successfully explained by differential Faraday rotation and depolarization due to $R M$ gradients. The gradients in $R M$ can also underlie the depolarization observed in the NE part of the galaxy.

None of the modeled processes predict an enhanced level of depolarization in the regions of both outer magnetic arms in agreement with observations. In fact, a completely different set of data at $1.43 \mathrm{GHz}$ shows that all those regions are still polarized at this frequency (Fig. 4 in Paper I) and must be Faraday-thin, thus avoiding strong Faraday effects. As both outer magnetic arms manifest depolarization properties similar to the $N$ inner magnetic arm, they cannot arise from reduced depolarization. Therefore, outer magnetic arms require an additional process that enhances regular component of the magnetic field.

\subsection{Modeling of magnetic field components}

In Sect. 3.2, we demonstrated that the outer magnetic arms in NGC 4254 involve a statistically more regular magnetic field than the other galaxy regions with a comparable level of starforming activity. We keep in mind that the regular field strength derived from the polarized intensity (Sect. 2.1) contains coherent and anisotropic components. Gas compression and stretching (with shearing) are well-recognized processes that can modify the coherent field and produce anisotropic field from isotropic (random) one (Sokoloff et al. 1998; Beck et al. 2005). In Paper I, we suggested that the southern polarized ridge may indeed be affected by stretching/shearing forces of tidal origin. As less likely, but still possible, we considered compressional forces acting in the southern disk portion due to the ram pressure of hot cluster gas. We propose here an analytical model to discriminate between stretching and ram-pressure alternatives as well as to explain the origin of outer magnetic arms.

In this model, we describe the galactic magnetic field in the cylindrical polar coordinates $(R, \phi, z)$ with $(R, \phi)$ aligned with the galaxy plane and with azimuthal angle $\phi$ counted counterclockwise from the northern edge of the galaxy major axis. The observer (sky) plane is inclined to the galactic plane by the galaxy inclination angle $i=42^{\circ}$ (Paper I). The discussed compression hypothesis involves external processes to act only in the outskirts of the galaxy, while the western outer magnetic arm, which is inside the galaxy disk, would remain unexplained. Therefore, we concentrate on modeling the southern magnetic arm.

As the initial properties of the modeled southern arm (without stretching or compression) we take the magnetic properties of the $N$ arm (Table 2), which we expect to be undistorted by tidal (stretching) or ram-pressure (compression) forces and to contain no anisotropic field. We notice that in the $N$ arm (exactly region 3 , Table 2) magnetic field vectors are aligned very close with the direction of the galaxy major axis (the difference is about $10^{\circ}$, Fig. 4). In the southern magnetic arm (region 1 , Table 2) they are oriented almost perpendicularly to the galaxy minor axis (difference of about $12^{\circ}$ ). Therefore, without any correction for projection effects we can approximate the coherent field in the southern arm by its azimuthal component only of strength equal to the regular field observed in the $N$ arm. Hence, following the approach of Sokoloff et al. (1998) the components of magnetic field $\boldsymbol{B}_{1}$ in the modeled southern arm are initially:

$$
\begin{aligned}
B_{\phi, 1} & =\bar{B}+b_{\phi, 1} \\
B_{R, 1} & =b_{R, 1} \\
B_{z, 1} & =b_{z, 1}
\end{aligned}
$$

where $\bar{B}$ denotes volume (ensemble) averaged magnetic field, thus its coherent part $\bar{B}=\left\langle B_{\phi, 1}\right\rangle$ and $b_{\phi, 1}, b_{R, 1}, b_{z, 1}$ are random magnetic field components, with $\left\langle b_{\phi, 1}\right\rangle=\left\langle b_{R, 1}\right\rangle=\left\langle b_{z, 1}\right\rangle=0$. Assuming at the beginning a fully isotropic random field, we 
Table 5. Modeling of the southern magnetic arm of NGC 4254 by stretching/shearing and compression processes. The radio properties of the $N$ magnetic arm are regarded as the initial conditions for the $S$ magnetic arm (region 3 and 1 in Table 2, respectively). The compression and stretching factors were adjusted so as to yield the observational values (given in italics) of synchrotron emissivity ratio $S_{2} / S_{2}$ or the degree of polarization in the southern arm $p_{2}$ (see Sect. 3.4).

\begin{tabular}{lccc}
\hline \hline & $\begin{array}{c}\text { stretching } \eta \text { or } \\
\text { compression } \zeta \text { factor }\end{array}$ & $S_{2} / S_{1}$ & $p_{2}$ \\
\hline Observed & - & $2.0 \pm 0.2$ & $0.37 \pm 0.03$ \\
Stretching & 1.53 & 2.0 & 0.39 \\
Stretching & 1.47 & 1.8 & 0.37 \\
Compression & 1.24 & 2.0 & 0.29 \\
Compression & 1.84 & 8.1 & 0.37 \\
\hline
\end{tabular}

demand $\left\langle b_{\phi, 1}^{2}\right\rangle=\left\langle b_{R, 1}^{2}\right\rangle=\left\langle b_{z, 1}^{2}\right\rangle=\sigma^{2}$, where $\sigma$ is the standard deviation of a random field in one dimension.

We assume that the stretching/shearing is along the southern magnetic arm. This should keep constant the strength of the initial coherent field and produce an excess in the form of azimuthal random component in the azimuthal direction, thus providing for the field anisotropy. We approximately scale the strength of this process by a single stretching factor $\eta$, giving the field components in response to stretching in the form:

$B_{\phi, 2}=\bar{B}+\eta b_{\phi, 1}$

$B_{R, 2}=b_{R, 1}$

$B_{z, 2}=b_{z, 1}$.

In our approach, $\eta=1$ means no stretching/shearing, which corresponds to the shear strength $K=0$ in the work of Beck et al. (2005).

With these magnetic field strengths, we can derive two nondimensional quantities in the observer frame: the ratio $S_{2} / S_{1}$ of the observed total synchrotron intensities from the southern arm after $\left(S_{2}\right)$ and before stretching $\left(S_{1}\right)$; and the degree of polarization $p_{2}$ of synchrotron emission after stretching. In these derivations, we generally follow the approach of Sokoloff et al. (1998) for the case of magnetic field and cosmic ray energy equipartition. In this case, the number density of CRs $N_{\mathrm{CR}}$ scales with the magnetic field as $B^{2}$. However, due to the CRs diffusion, we do not expect a complete spatial correlation between both quantities to hold locally, i.e., on scales below $1 \mathrm{kpc}$ (cf. discussion in Beck et al. 2003). We then treat both the quantities as locally statistically independent variables whose ensemble averages satisfy $\left\langle N_{\mathrm{CR}}\right\rangle \propto\left\langle B^{2}\right\rangle$. Under this condition we get:

$\frac{S_{2}}{S_{1}}=\frac{\left\langle B_{2}^{2}\right\rangle\left\langle B_{\perp, 2}^{2}\right\rangle}{\left\langle B_{1}^{2}\right\rangle\left\langle B_{\perp, 1}^{2}\right\rangle}=\frac{\left[\bar{B}^{2}+\sigma^{2}\left(\eta^{2}+2\right)\right]\left[\bar{B}^{2}+\sigma^{2}\left(\eta^{2}+1\right)\right]}{\left(\bar{B}^{2}+3 \sigma^{2}\right)\left(\bar{B}^{2}+2 \sigma^{2}\right)}$

where $B_{\perp, 1}$ and $B_{\perp, 2}$ are perpendicular to the line of sight components of the magnetic field in the southern arm before and after stretching, respectively, and $B_{1}$ and $B_{2}$ are the corresponding total field strengths.

For the degree of polarization after stretching we obtain:

$p_{2}=p_{0} \frac{\bar{B}^{2}+\left(\eta^{2}-1\right) \sigma^{2}}{\bar{B}^{2}+\left(\eta^{2}+1\right) \sigma^{2}}$

where $p_{0} \approx 0.75$ is the maximum degree of polarization. We notice that $S_{2} / S_{1}$ as well as $p_{2}$ do not depend on the galaxy inclination, as expected for the orientation of magnetic vectors in the southern arm, perpendicular to the minor axis.

In our modeling of the southern arm, we adjust the stretching factor $\eta$ to get exactly the observed value of the ratio $S_{2} / S_{1}$ (given in Table 5) and then compare the modeled $p_{2}$ to the observed value (Table 5). In the second approach, we adjust $\eta$ to get for $p_{2}$ its observational value and then we compare modeled $S_{2} / S_{1}$ to the actual value. The results presented in Table 5 show that the modeled values fit very well the observed ones within their uncertainties. So a tidal stretching can very effectively produce the anisotropic magnetic field in NGC 4254 . About $60 \%$ of the observed polarized intensity of the southern magnetic arm comes in this case from the anisotropic random field. The determined stretching factor $\eta \approx 1.5$ is rather small when compared, e.g., to the typical shear strengths in strongly-barred galaxies (about 10 in NGC 1097, Beck et al. 2005).

The second process - compression - would produce enhanced magnetic field components tangent to the compression plane, leaving the component normal to it unchanged (see Beck et al. 2005). We suspect that in the case of NGC 4254, compression might act in the plane perpendicular to the galaxy disk, from the southeastern side of it. We assume that due to compression the tangent regular and random field components are $\zeta$ times stronger than the original ones. This compression factor can be interpreted as the ratio of gas densities $\left(\zeta=\rho_{2} / \rho_{2}\right)$ before $\left(\rho_{1}\right)$ and after compression $\left(\rho_{2}\right)$. Hence, the magnetic field in the southern arm after compression is:

$$
\begin{aligned}
B_{\phi, 2} & =\zeta \bar{B}+\zeta b_{\phi, 1} \\
B_{R, 2} & =b_{R, 1} \\
B_{z, 2} & =\zeta b_{z, 1} .
\end{aligned}
$$

Similar to the analysis of stretching, we derive in case of compression, the synchrotron emission ratio:

$$
\begin{aligned}
\frac{S_{2}}{S_{1}}= & \frac{\left\langle B_{2}^{2}\right\rangle\left\langle B_{\perp, 2}^{2}\right\rangle}{\left\langle B_{1}^{2}\right\rangle\left\langle B_{\perp, 1}^{2}\right\rangle}=\frac{\left[\zeta^{2} \bar{B}^{2}+\sigma^{2}\left(2 \zeta^{2}+1\right)\right]}{\left(\bar{B}^{2}+3 \sigma^{2}\right)} \\
& \times \frac{\left\{\zeta^{2} \bar{B}^{2}+\sigma^{2}\left[\zeta^{2}\left(1+\sin ^{2} i\right)+\cos ^{2} i\right]\right\}}{\left(\bar{B}^{2}+2 \sigma^{2}\right)}
\end{aligned}
$$

and the degree of polarization in the southern arm:

$$
p_{2}=p_{0} \frac{\zeta^{2} \bar{B}^{2}+\sigma^{2}\left(\zeta^{2}-1\right) \cos ^{2} i}{\zeta^{2} \bar{B}^{2}+\sigma^{2}\left[\zeta^{2}\left(1+\sin ^{2} i\right)+\cos ^{2} i\right]} .
$$

According to the above expressions, we model that $\zeta \approx 1.2$ gives a synchrotron emissivity ratio $S_{2} / S_{1}$ equal to the observed value (Table 5), but the corresponding degree of polarization of 0.29 is lower than the observed one $(0.37 \pm 0.03)$. A higher compression value, which yields a stronger anisotropic field and a sufficient degree of polarization, results in a synchrotron emission ratio (8.1) much higher than the one actually observed (2.0 \pm 0.2$)$. In comparison to the stretching process, for the same production of anisotropic field, compression produces a larger amount of isotropic random field, resulting in a lower degree of the modeled polarization, which is not consistent with observations.

We checked that our conclusions are not too sensitive to the galaxy orientation: lowering the galaxy inclination by 2 degrees (to $i=40^{\circ}$ ) enhances the degree of polarization by only $1 \%$. We also performed an analysis of compression in two other cases: the equipartition assumption with a full local spatial correlation between the density of CRs and the magnetic field energy, 
and the case of no equipartition, i.e., constant density of CRs. Although we regard these assumptions as less probable than those actually adopted (no local correlation), we mention that in the case of constant density of CRs for the value of $\zeta=1.5$, the synchrotron emission ratio $S_{2} / S_{1}$ attains the observed value and the degree of polarization reaches $p_{2}=0.33$, which is quite consistent with the observed $p_{2}=0.37 \pm 0.03$. However, the higher compression amount $(\zeta=1.8)$, while yielding a sufficient degree of polarization, results in the ratio $S_{2} / S_{1}=2.9$, inconsistent with observations. The case of equipartition with local spatial correlation gives similar results. The reason why different assumptions on equipartition (with and without local correlation) give close results in model fitting is the low factor $\zeta$ that is needed to explain the enhancement of the magnetic field in the southern arm of NGC 4254. Due to nonlinear influence of $\zeta$ on synchrotron emission ratio (Eq. (14)), much larger differences in results are expected for shocks with larger $\zeta$. Such cases (with full local spatial correlation) were studied by Beck et al. (2005), see their Fig. 21 for a rough comparison.

We conclude that the stretching hypothesis does likely provide a proper explanation for the high-field regularity in the southern magnetic arm.

\subsection{The influence of the cluster environments}

One of the most characteristic features of magnetic structure in NGC 4254 is the well-aligned magnetic field in the two outer (southern and western) magnetic arms. Although, on the whole, the field regularity seems to be dominated by the production of random fields in star-forming regions (Sect. 3.2), in outer magnetic arms the regularity attains larger values than in other disk regions of similar SFR, and thus of similar random fields.

According to our analysis of depolarization (Sect. 3.3), the outer magnetic arms are marginally depolarized, likewise the $N$ arm, and their high magnetic regularity cannot result from a reduced depolarization. This conclusion corresponds with similar results on magnetic arms in NGC 6946 (Beck 2007). However, in the case of NGC 4254, two strong (outer) magnetic arms are located downstream of nearby density waves, which was not observed in other galaxies. Even the northern part of NGC 4254 shows (inner) magnetic arms on the upstream side of spiral arms. It appears that the unusual properties of outer magnetic arms in NGC 4254 are instead associated with some external forces and the cluster environment.

Using the modeling of magnetic field components in the southern magnetic arm (Sect. 3.4), we have shown that the compression due to the external ram-pressure of hot cluster gas does not provide a sufficiently-high magnetic field regularity for the observed level of polarized emission. In Paper I, we also enumerated other difficulties of this idea, e.g., as low depletion of H I gas and globally-enhanced, instead of spatially-truncated, star formation in NGC 4254.

There are known examples of galaxies with a distinct action of strong compressional forces and enhanced magnetic fields. Such forces were argued to be present in the barred galaxies (Beck et al. 2005), and assessed by the density ratio of pre- and post-shock gas. We searched for such a compressional evidence in the contrast ratio of polarized, nonthermal, and $\mathrm{HI}$ emission in NGC 4254, measured between magnetic or optical arms and their outside locations separated by 1.5 beams. We inspected five such positions indicated in Fig. 1, and present the results in Table 6 . The polarized ridge (locations $\mathrm{a}$ and $\mathrm{b}$ ) and the $\mathrm{W}$ outer magnetic arm (c) do not reveal any distinctly large change in the analyzed distributions as compared with the other regions.
Table 6. Contrast ratio in and out of the spiral arms in NGC 4254 in polarized and total synchrotron as well as $\mathrm{HI}$ emission. The positions of the inspected five regions are denoted in Fig. 1.

\begin{tabular}{lrrrrr}
\hline \hline & $a$ & $b$ & $c$ & $d$ & $e$ \\
\hline Polarized intensity & 5.3 & 6.1 & 12.9 & 1.9 & 16.6 \\
Nonthermal intensity & 3.7 & 4.2 & 1.8 & 2.7 & 3.3 \\
H I emission & 2.1 & 1.8 & 2.3 & 2.5 & 1.4 \\
\hline
\end{tabular}

In view of the data currently available and the performed modeling of magnetic field components (Sect. 3.4), we suggest that the most likely hypothesis for the enhanced regular field in the outer magnetic arms are stretching forces of gravitational (tidal) origin. This prediction can be further checked by $R M$ data, not sensitive to anisotropic field. The $R M$ analysis cannot be performed for the southern outer magnetic arm (region 1), since the regular field component lies almost in the plane of the sky. However, as the strengths of different components of magnetic field for both the outer arms are very similar (Table 2), we suspect that both the arms are enriched in an anisotropic field of similar magnitude and origin. Hence, we perform $R M$ analysis for the western magnetic arm. The line-of-sight component of coherent magnetic field $\left\langle B_{\mathrm{coh} \|}\right\rangle$ can be estimated from the actual value of rotation measure:

$R M=0.812\left\langle B_{\mathrm{coh} \|}\right\rangle N_{\mathrm{e}} L$.

The mean thermal electron density $N_{\mathrm{e}}$ over the galaxy thermal disk pathlength $L$ can be estimated from the thermal radio emission (the emission measure $E M$ ), the fraction of the diffuse gas in the total ionized gas (see Sect.3.3), and the filling factor $f$. The inner $N$ magnetic arm (region 3, Fig. 5b) has the observed $R M=-43 \pm 17 \mathrm{rad} \mathrm{m}^{-2}$. Supposing for $N$ and $W$ magnetic arms the same values for $L, f$ and for the fraction of the diffuse gas, we get from (16) a prediction of rotation measure in the $\mathrm{W}$ arm (region 2) of about $150 \mathrm{rad} \mathrm{m}^{-2}$, assuming that the entire regular field in that region $(12.3 \mu \mathrm{G})$ is coherent. This is much more than the observed $+40 \pm 17 \mathrm{rad} \mathrm{m}^{-2}$. Even taking into account large statistical uncertainties in the measured $R M$ values, this disagreement can suggest that the polarized emission in the outer western arm comes not only from the regular coherent field traced by $R M$ data, but to a large extent from an anisotropic field, in agreement with the modeling of stretching effect.

The stretching and shearing origin of the high magnetic regularity in outer magnetic arms is also in accord with the study of magnetic field orientations (Sect. 3.1) indicating a strong influence of gaseous streamlines on the entire magnetic structure in that galaxy. In the Antennae merging system, where such stretching forces are expected in the NE portion of NGC 4038, at the base of the tidal tail, there is indeed a polarized ridge (Chyży \& Beck 2004) of high field regularity and with magnetic field vectors aligned with local gas flows.

Recently, Kantharia et al. (2008) detected the radio envelope of NGC 4254 at frequencies down to $240 \mathrm{MHz}$. They argue that the envelope is a signature of the ram-pressure gas, stripped from the galactic disk and now expanding. However, radio envelopes were also observed around non-cluster spirals (cf. Paper I) and their existence cannot be used in favor of ram-pressure effects. The argument that the envelope has a steep synchrotron spectrum $\left(\alpha_{\text {nth }}<-1.0\right)$ is not convincing either, as field galaxies also show spectral steepening at the disk outskirts, which results from CRs diffusion and energy losses (Sect. 3.2). Besides, comparing the ram pressure with the magnetic field pressure, they assumed 
several times lower magnetic field strength $(3 \mu \mathrm{G})$ than is actually observed (Fig. 5a). Next, their argument that the high starformation in NGC 4254 is due to ICM wind is surprising as the Virgo Cluster spirals show the opposite effect: a lower star formation for galaxies that experienced ICM-ISM stripping events (Paper I, Koopmann \& Kenney 2004).

Although NGC 4254 seems to be a "young" Virgo Cluster member (Paper I), it reveals distortions that instead of a ram pressure, they could be attributed to a gravitational harassment (Moore et al. 1996). It is visible in the galaxy's perturbed spiral pattern, disturbed H I emission, and enhanced level of star formation. We also propose that the enhanced total and anisotropic magnetic field, the strong southern polarized ridge, and the complex magnetic field morphology, not found in the field galaxies, could also be the manifestation of the harassment process. We expect that the dramatic morphological transformations of gas, as well as magnetic field, are still ahead of this object, when it is going to pass the Virgo Cluster core, and will experience severe ram pressure stripping and global tides.

Recent observations of other Virgo spirals (Vollmer et al. 2007; Weżgowiec et al. 2007) show that asymmetrical polarized emission are common among Virgo Cluster galaxies. The modeling of magnetic field components as applied to NGC 4254 in Sect. 3.4 can also be performed for those galaxies. This could at last allow for a statistical analysis of diverse influence of ICM on magnetic field structures in cluster spirals.

\section{Summary and conclusions}

We present the first detailed investigation of magnetic field, Faraday rotation measures, and depolarization of synchrotron emission in a spiral galaxy - NGC 4254 - embedded in the Virgo Cluster medium. In Paper I, we described our radio (VLA and Effelsberg) and X-ray (XMM-Newton) observations and data reduction, along with a comprehensive discussion of the galaxy's properties in different wavelengths. The complex polarization properties discovered in different regions of the galaxy are analyzed here, with the help of the newly developed concept of presenting magnetic field in the form of various "magnetic maps" (Sect. 2.3) and with analytical modeling of Faraday effects (Sect. 3.3), as well as modeling of compression and stretching forces on different magnetic field components (Sect. 3.4).

The performed study enabled us to obtain the following results:

- The distribution of $R M$ in NGC 4254 reveals large areas of coherent (unidirectional) magnetic field, which resemble a perturbed axisymmetric dynamo mode or a mixture of axisymmetric and bisymmetric modes. The magnetic field vectors are oriented outwards from the galaxy disk, contrary to other galaxies.

- The constructed "magnetic maps" show that the total magnetic field in NGC 4254 is stronger than in typical field spirals, reaching $17 \mu \mathrm{G}$ in the interarm regions, $22 \mu \mathrm{G}$ in spiral arms, and even $25 \mu \mathrm{G}$ in the disk core (assuming $1 \mathrm{kpc}$ disk thickness). The magnetic energy exceeds the thermal one in almost all regions of the galaxy disk. It surpass even the turbulent gas energy in the southern polarized ridge, the western interarm region, and at the disk outskirts, and may become dynamically important.

- The regular field strength reaches the largest value of $13 \mu \mathrm{G}$ and the highest field regularity of about 0.8 in the two (southern and western) outer magnetic arms. The inner $\mathrm{N}$ magnetic arm involves a weaker regular field $(8 \mu \mathrm{G})$ and less field regularity (0.5). While the strongest regular field occurs in magnetic arms, the total and random field strengths are at the highest in the optical arms.

- The magnetic field vectors, of which the Faraday-free pitch angles change throughout the galaxy from zero (in the polarized ridge) to more than $40^{\circ}$ (in the north), always keep their orientation close to the optical filaments (within $20^{\circ}$ ). The dynamo-generated magnetic fields must be significantly modified in NGC 4254 by density waves and gas flows.

- The distribution of depolarization cannot be easily explained by any individual process (Sect. 2.1), and thus various Faraday effects must be at work in different galaxy regions. Our modeling demonstrated (Sect. 3.3) the dominant role of Faraday dispersion and differential Faraday rotation within the spiral arms and in the galaxy center, where the observed depolarization is most significant $(D P \approx 0.8-0.6)$. None of the modeled Faraday depolarization effects influence the outer or inner magnetic arms, which is in agreement with the observed depolarization there.

- The strength of total magnetic field does correlate very well with the star-formation rate of individual regions in the galaxy disk (the correlation coefficient $r=0.95$ ), giving a well-fitted power-law relation with a slope $+0.18 \pm 0.01$. However, in the same regions, the regularity of magnetic field shows a high level of anticorrelation $(r=-0.67)$ with the star formation activity, which mainly results from efficient production of random field in vivid star-forming regions. For the first time, we show that this relation is well approximated by a power-law with the slope of $-0.32 \pm 0.01$. The enhanced field regularity in the outer magnetic arms are, however, not fully controlled by the star formation.

- Our modeling of magnetic field components in magnetic arms indicates that the enhanced magnetic field regularity in the outer magnetic arms can be produced by stretching and shearing forces, likely of tidal origin, which produce an anisotropic magnetic component and enhance polarized emission. This is supported by the analysis of depolarization and Faraday rotation measures, while being in accord with the field regularity-SFR relation. Hence, modeling of magnetic field components seems to be a great tool to discriminate between different physical phenomena acting in cluster environment.

The validity of the proposed stretching origin of enhanced magnetic field in the outer arms of NGC 4254 could be further explored by some advanced MHD modeling, able to discriminate between magnetic field components and reproduce the perturbed structure of the galactic spiral arms. This kind of modeling of magnetic field components performed for NGC 4254 can also be applied to other Virgo Cluster spirals and would allow for a statistical analysis of the magnetic field evolution within galaxies influenced by the cluster medium.

Acknowledgements. I would like to thank Dr Rainer Beck and Prof. Marek Urbanik for helpful discussions and stimulating comments, especially on depolarization effects. This work was supported by the Polish Ministry of Science and Higher Education, grant 2693/H03/2006/31.

\section{References}

Beck, R. 2007, A\&A, 470, 539

Beck, R., \& Hoernes, P. 1996, Nature, 379, 47

Beck, R., Brandenburg, A., Moss, D., Shukurov, A., \& Sokoloff, D. 1996, ARA\&A, 34, 155

Beck, R., Shukurov, A., Sokoloff, D., \& Wielebinski, R. 2003, A\&A, 411, 99

Beck, R., Fletcher, A., Shukurov, A., et al. 2005, A\&A, 444, 739 
Burn, R. 1966, MNRAS, 133,67

Cayatte, V., van Gorkom, J. H., Balkowski, C., \& Kotanyi, C. 1990, AJ, 100, 604 Chyży, K. T., \& Beck, R. 2004, A\&A, 417, 541

Chyży, K. T., Ehle, M., \& Beck, R. 2007, A\&A 474, 415

de Avillez, M. A., \& Breitschwerdt, D. 2005, ApJ, 634, L65

Elstner, D., Otmianowska-Mazur, K., von Linden, S., \& Urbanik, M. 2000, A\&A, 357, 129

Elstner, D. 2005, in The Magnetized Plasma in Galaxy Evolution, ed. K. T. Chyży et al. (Krakow: OAUJ), 117

Ehle, M., \& Beck, R. 1993, A\&A, 273, 45

Fletcher, A., Berkhuijsen, E. M., Beck, R., \& Shukurov, A. 2004a, A\&A, 414, 53

Fletcher, A., Beck, R., Berkhuijsen, E. M., Horellou, C., \& Shukurov, A. 2004b, in How does the galaxy work?, ed. W. J. Alfaro et al. (Dordrecht: Kluwer), 299

Jansen, F., Lumb, D., Altieri, B., et al. 2001, A\&A, 365, L1

Johnston-Hollitt, M., Hollitt, C. P., \& Ekers, R. D. 2004, The Magnetized Interstellar Medium, ed. B. Uyaniker et al. (Katlenburg: Copernicus), 13

Kantharia, N. G., Rao, A. P., \& Sirothia, S. K. 2008, MNRAS, 381, 173

Knapen, J. H., Stedman, S., Bramich, D. M., Folkes, S. F., \& Bradley, T. R. 2004, A\&A, 426, 1135

Koopmann, R. A., \& Kenney, J. D. P. 2004, ApJ, 613, 851

Krause, F., \& Beck, R. 1998, A\&A 335, 789

Moore, B., Kartz, N., Lake, G., Dressler, A., \& Omler, A., Jr. 1996, Nature, 379, 613
Moss, D., Shukurov, A., Sokoloff, D., Beck, R., \& Fletcher, A. 2001, A\&A, 380, 55

Moss, D., Snodin, A. P., Englmaier, P., et al. 2007, A\&A, 465, 157

Patrikeev, I., Fletcher, A., Stepanov, R., et al. 2006, A\&A 458, 441

Phookun, B., Vogel, S. N., \& Mundy, L. G. 1993, ApJ, 418, 113

Rand, R. J., \& Kulkarni, S. T. 1989, ApJ, 343,760

Roediger, E., \& Hensler, G. 2005, A\&A, 433, 875

Rohde, R., Elstner, D., \& Rümldiger, G. 1997, Acta Cosmologica, 23, 205

Rohde, R., Beck, R., \& Elstner, D. 1999, A\&A, 350, 423

Shukurov, A. 1998, MNRAS, 299, L21

Shukurov, A. 2005, in Cosmic Magnetic Fields, ed. R. Wielebinski, \& R. Beck (Heidelberg: Springer), 111

Sofue, Y., Koda, J., Nakanishi, H., \& Hidaka, M. 2003, PASJ, 55, 75

Sokoloff, D. D., Bykov, A. A., Shukurov, A., et al. 1998, MNRAS, 299,189

Soida, M., Urbanik, M., \& Beck, R. 1996, A\&A, 312, 409

Soida, M., Otmianowska-Mazur, K., Chyży, K., \& Vollmer, B. 2006, A\&A, 458, 727

Theil, H. 1972, Statistical Decomposition Analysis (Amsterdam/London: NorthHolland)

Vollmer, B., Soida, M., Beck, R., et al. 2007, A\&A, 464, L37

Walterbos, R. A. M. 2000, in The Interstellar Medium in M 31 and M33, ed. E. M. Berkhuijsen et al. (Shaker, Aachen), 99

Weżgowiec, M., Urbanik, M., Vollmer, B., et al. 2007, A\&A, 471, 93

Widrow, L. M. 2002, Rev. Mod. Phys., 74, 775 